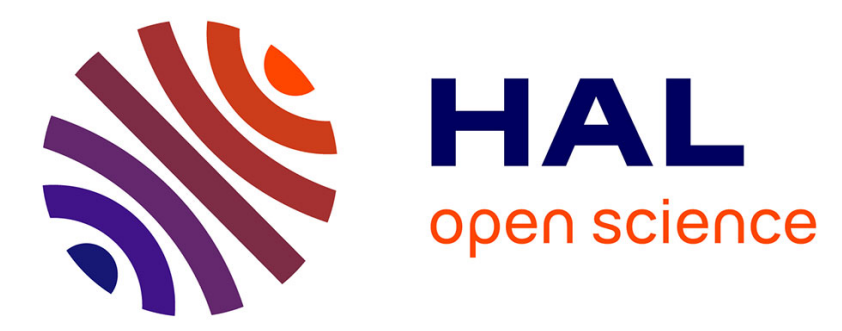

\title{
The many roles of myosins in filopodia, microvilli and stereocilia
}

\author{
Anne Houdusse, Margaret A Titus
}

\section{To cite this version:}

Anne Houdusse, Margaret A Titus. The many roles of myosins in filopodia, microvilli and stereocilia. Current Biology - CB, 2021, 31 (10), pp.R586-R602. 10.1016/j.cub.2021.04.005 . hal-03281243

\section{HAL Id: hal-03281243 \\ https://hal.sorbonne-universite.fr/hal-03281243}

Submitted on 8 Jul 2021

HAL is a multi-disciplinary open access archive for the deposit and dissemination of scientific research documents, whether they are published or not. The documents may come from teaching and research institutions in France or abroad, or from public or private research centers.
L'archive ouverte pluridisciplinaire HAL, est destinée au dépôt et à la diffusion de documents scientifiques de niveau recherche, publiés ou non, émanant des établissements d'enseignement et de recherche français ou étrangers, des laboratoires publics ou privés. 


\section{REVIEW}

The many roles of myosins in filopodia, microvilli and stereocilia

Anne Houdusse ${ }^{1, *}$ and Margaret A. Titus ${ }^{2, *}$

${ }^{1}$ Structural Motility, Institut Curie, Paris Université Sciences et Lettres, Sorbonne Université, CNRS UMR144, 75005 Paris, France

${ }^{2}$ Department of Genetics, Cell Biology, and Development, University of Minnesota, Minneapolis, MN 55455, USA

*Correspondence: Anne.Houdusse@curie.fr (A.H.); titus004@umn.edu (M.A.T.)

\section{Summary}

Filopodia, microvilli and stereocilia represent an important group of plasma membrane protrusions (AU:OK?). These specialized projections are supported by parallel bundles of actin filaments and have critical roles in sensing the external environment, increasing cell surface area, and acting as mechanosensors. While actin-associated proteins are essential for actin-filament elongation and bundling in these protrusions (AU:OK?), myosin motors have a surprising role in the formation and extension of filopodia and stereocilia and in the organization of microvilli. Actin regulators and specific myosins collaborate in controlling the length of these structures. Myosins can transport cargoes along the length of these protrusions, and, in the case of stereocilia and microvilli, interactions with adaptors and cargoes can also serve to anchor adhesion receptors to the actin-rich core via functionally conserved motor-adaptor complexes (AU:OK?). This review highlights recent progress in understanding the diverse roles myosins play in filopodia, microvilli and stereocilia.

\section{Introduction}

Filopodia, microvilli and stereocilia constitute a special class of slender membrane protrusions supported by bundles of crosslinked parallel actin filaments; these 
protrusions are used by cells to sense and interact with their environments (Figure 1). The actin filaments are organized with their fast-growing (plus or barbed) ends oriented towards the tip and the slower-growing (minus or pointed) end embedded in the actin cortex. Filopodia are highly dynamic and found in organisms (AU:OK?) from across the eukaryotic tree, including Rhizaria, Discoba, Apusozoa, Amoebozoa and Holozoa, and thus can be considered as the most ancient of this group of actin-based protrusions ${ }^{1-4}$. Unicellular organisms employ filopodia to physically interact with the environment, mediate substrate adhesion during migration, and capture prey ${ }^{5,6}$. While microvilli are present throughout Holozoa, they appear first in unicellular relatives of Metazoa, most notably in choanoflagellates where they make up the microvillar collar that plays a role in feeding ${ }^{7,8}$. Microvilli provide epithelia with increased surface area for absorption and also serve as a protective barrier ${ }^{9}$. Stereocilia are almost exclusively found in vertebrates where they have essential roles in balance and hearing ${ }^{8}$. Microvilli and stereocilia are tightly linked together by cadherin-based linkages and their lengths are subject to remarkable control. Microvilli have a uniform length that varies between cell types, whereas stereocilia are graded in height ${ }^{9,10}$. Each of these projections emerges from the dense, actin-rich cortical cytoskeleton through the action of actin polymerization factors and crosslinkers that work in concert to initiate, extend and bundle the core parallel actin filaments. A good deal of attention has been paid to the role of the actin regulators in building filopodia, microvilli and stereocilia, but it is now appreciated that myosin motors also have critical roles in both the formation and function of these special structures (Figure 2).

Myosins are a diverse family of actin-based motors found in all major eukaryotic lineages; they can drive intracellular transport, promote contractility, organize actinbased structures, generate tension and aid DNA repair, to name a few functions ${ }^{11-15}$. Myosins can be classified as processive motors, strain-sensitive anchors or tethers, or 
contractile motors ${ }^{14}$. The function of a given myosin is dictated by a combination of the intrinsic kinetic properties of the conserved motor domain (typically referred to as the 'head') and the interaction of the myosin with partner proteins determined by various domains present in its carboxy-terminal tail. The tail also dictates whether the myosin is a monomer, dimer or multimer. A related group of myosins - termed the MyTH4FERM (MF; myosin tail homology - band 4.1, ezrin, radixin, moesin) myosins - make significant contributions to the formation and function of filopodia, microvilli and stereocilia. These myosins are characterized by the presence of one (MYO10) or two (MYO7, MYO15) MF domains in their carboxy-terminal tail region that bind to distinct partner proteins required for either transport or anchoring membrane protein complexes ${ }^{16}$. The widespread use of MF myosins to build thin actin-based protrusions reflects both the deep evolutionary conservation of this family of myosins as well as the likely ancient origin of these structures themselves ${ }^{3,8}$. This review summarizes what is currently known about the pivotal role of myosin motors in the formation and function of filopodia, microvilli and stereocilia.

\section{Myosins in filopodia formation and function}

Filopodia are highly dynamic structures that can vary in length from two to up to hundreds of micrometers and can be stabilized by adhesion to neighboring cells or the substrate ${ }^{17-19}$. They play important roles in cellular processes such as adhesion to the extracellular matrix (ECM), guidance towards chemoattractants in development, wound healing and viral infection ${ }^{17,20,21}$. Key filopodial proteins and adhesion receptors are present at the tip, where they mediate substrate attachment ${ }^{22}$. Filopodia emerge from the cell cortex, which is composed of a branched dendritic network of actin filaments mostly nucleated by the Arp2/3 complex. The overall mechanism of filopodia initiation and extension is highly conserved. Initiation requires activation of a small GTPase (such as (dc42), as well as actin regulators (such as Ena/VASP), an actin regulator (AU:OK?) 
with both anti-capping and bundling activity, and/or formins, which nucleate the formation and extension of parallel actin filaments ${ }^{23}$. Filaments are then either reorganized from the cortical network or nucleated de novo so that they are oriented perpendicular to the membrane ${ }^{23}$ (Figure 3A). A cluster of 10-15 actin filaments is minimally required to overcome membrane tension to push the membrane outwards ${ }^{24}$. Filament extension by Ena/VASP or formins is accompanied by rapid actin crosslinking by proteins such as fascin in mammalian cells. While thin protrusions made by cells are generally all designated as filopodia, it should be noted that filopodia are a diverse group of structures that can be initiated by different mechanisms and have distinctive functions.

\section{Filopodial initiation}

Two phylogenetically distinct MF myosins are required for filopodia initiation and extension - MYO10 in mammalian cells and DdMyo7 in Amoebozoa (AU: should this just be referred to as Myo7 rather than DdMyo7, as you follow this with "in Amoebozoa"? Or should it say "DdMyo7 in Dictyostelium"? ${ }^{5,25}$. These myosins cluster on the membrane to form initiation sites and localize to the tips of growing filopodia. MYO10 consists of a motor domain followed by a semi-rigid lever arm region that amplifies the swing of the motor as it moves along actin. The lever arm consists of three light-chain-binding IQ motifs that bind calmodulin or a calmodulin-like light chain, and an elongated stable $\alpha$-helix. The lever arm is followed by a dimerization domain that dictates the formation of an anti-parallel dimer ${ }^{26-28}$. The MYO10 tail contains three pleckstrin homology $(\mathrm{PH})$ domains that target MYO10 to phosphatidylinositol $(3,4,5)$ trisphosphate $\left(\mathrm{PIP}_{3}\right)$-rich regions of the plasma membrane, followed by a carboxyterminal MF domain that interacts with various partners, including integrins. The tail of amoeboid DdMyo7, in contrast, contains two MF domains separated by an SH3 domain. Interestingly, functional studies with MYO10 and DdMyo7 show that filopodia formation 
requires a motor domain, a post-lever arm region (AU: please clarify what you mean by "post-lever arm region") and a dimerization region 25,26,29,30. Furthermore, the MYO10 MF domain can functionally substitute for a DdMyo7 MF domain $25,29,30$. The finding that DdMyo7 and MYO10 share the same general operating principles is consistent with the mechanism of filopodial MF action being evolutionarily conserved.

The requirement for a dimerized motor for filopodia initiation is highlighted by the finding that a forced dimer of either the MYO10 or the DdMyo7 motor domain can drive filopodia formation ${ }^{29,31}$. These two forced dimers lack binding sites for partners, revealing the critical role of motor function during the initiation step. This does not mean that the tail is entirely dispensable. In the case of the DdMyo7 forced dimer, the resulting protrusions are shorter than wild-type filopodia ${ }^{29}$, indicating that the MF domains are needed for the recruitment of elongation factors (see below). An engineered dimeric motor based on MYO6 (MYO6+), a myosin motor (AU:OK?) that moves towards the plus end of actin filaments instead of the minus end, can also generate filopodia-like protrusions ${ }^{32}$. Unlike the MYO10 or DdMyo7 forced dimers, MYO6+ requires an interaction with endosomal membranes mediated by its binding partner GIPC, a PDZ adaptor protein, to generate filopodia-like protrusions, suggesting a different mode of action ${ }^{29,32}$.

Filopodia emerge from MYO10 foci close to nascent focal adhesions rich in actin, integrin and vinculin at the leading edge of the cell ${ }^{33,34}$. VASP is also present at these sites, where it could work in concert with MYO10 to reorganize Arp2/3-branched actin filaments to initiate filopodia. MYO10 and DdMyo7 are largely cytosolic and exist in an autoinhibited conformation mediated by interactions between the motor and tail regions, similar to other myosins ${ }^{35,36}$. Recruitment to the membrane or cortex relieves this autoinhibition, disrupting the head-tail interaction and inducing dimer 
formation ${ }^{29,36}$. The exact mechanism by which the head interacts with the tail and how membrane/cortical recruitment of the motor switches the monomer (AU:OK?) from a compact, closed state to an elongated dimer is not yet understood, although a role for $\mathrm{PIP}_{3}$ binding and the cortical actin network has been identified for MYO10 and DdMyo7, respectively ${ }^{35,37}$ (AU:OK?). Activation in local clusters favors dimerization of the motor at initiation sites where it can pull on nearby actin filaments, promoting the bundling of actin filaments. This would orient the filaments correctly with their plus ends abutting the membrane in a position favorable for growth into a filopodium (Figure 3A). The motors can bind to and walk up adjacent parallel actin filaments, zippering them together as they move up towards the plus ends. The actin filaments are then coalesced into a bundle of sufficient size to push against the membrane, in collaboration with VASP (and/or formin) ${ }^{38}$

\section{Filopodia elongation and adhesion}

The initial nucleation/initiation of a filopodium is followed by MYO10-dependent elongation, with MYO10 switching roles from an organizer of actin filaments at the membrane to a transporter (Figure 3B). MYO10 undergoes intrafilopodial motility and appears to transport VASP towards the filopodial tip ${ }^{38,39}$, where VASP serves to inhibit actin-filament capping and promote ongoing polymerization of the growing filaments. The kinetic and structural features of MYO10 are optimized for its function as a transporter on bundles 27,40 . Dimerized MYO10 is a processive motor that moves most efficiently along bundles of actin filaments at around $660 \mathrm{~nm} \mathrm{~s}^{-1}$ with an average run length of $2 \mu \mathrm{m}^{27}$. This velocity closely matches those of MYO10 moving along filopodia in cells $\left(580-840 \mathrm{~nm} \mathrm{~s}^{-1}\right)^{41}$. MYO10 is an anti-parallel dimer ${ }^{26,27}$ that can take variable, large steps (up to $58 \mathrm{~nm}$ ) preferentially along actin bundles because of both the positioning of the converter domain (AU: please could you clarify what the converter domain is) and extended, flexible lever arm as well as the atypical dimerization of this 
myosin. This gives MYO10 a more flattened orientation relative to the actin filament, enabling this myosin to step across actin filaments in a bundle and take larger steps as it translocates up the filopodium ${ }^{27}$. It should be noted that this structural adaptation is also advantageous for capturing irregularly organized actin filaments at the cortex during filopodia initiation. The overall organization and regulation of MYO10 compared with other processive motors such as MYO5 reveals how structural adaptations can tune these two motors for the most effective (AU:OK?) trafficking on distinct cellular $\operatorname{tracks}^{27,42}$.

Cells exploit filopodial dynamics to explore the environment; filopodia extend out ahead of the lamellipodium and provide initial contacts with the substrate or ECM via the tip (Figure 3B). Force transmitted along the length of the filopodium by MYH9 (MYO2A) located at the base leads to stabilization of filopodial contact with the $\mathrm{ECM}^{43}$. MYO10 is not only required for filopodia extension but also mediates cadherin- and integrin-based adhesion, binding to cadherin/integrin cytoplasmic tails via its carboxyterminal FERM domain and co-localizing with cadherins and integrins at filopodial tips $^{44,45}$. There is currently no direct evidence that MYO10 transports integrins along the length of the filopodial shaft. Rather, it seems that once MYO10 arrives at the tip, it switches from being a transporter to being an anchor that traps a small but critical population of integrins at the tip. Surprisingly, recent work has revealed that the MYO10 FERM domain also binds to the cytoplasmic tail of a-integrin and that binding to both $\alpha-$ and $\beta$-integrin tails is important for localized activation of integrins at the filopodial tip $^{46}$. MYO10 binding itself does not activate the integrin heterodimer because binding to both tails is likely to keep the heterodimer in a low-affinity conformation. Instead, it appears that the myosin anchors the integrin heterodimer at the tip, positioning it for activation by binding of the talin FERM domain (Figure 3B), possibly following the influx of $\mathrm{Ca}^{2+}$ by L-type channels and subsequent cleavage by calpain ${ }^{18,22,47}$. Displacement of 
MYO10 from the integrin tails by talin would free MYO10 to bind another integrin or to promote continued filopodia elongation. This displacement would result in adhesion sites forming within the shaft of these specially stabilized filopodia. The mechanism by which the MYO10 filopodial tip complex controls actin polymerization and adhesion in a tension-dependent manner and the composition of the tip itself remain to be characterized.

The essential role of MYO10 in filopodia formation led to the suggestion that deletion of this motor would be lethal for a multicellular organism. A large fraction of

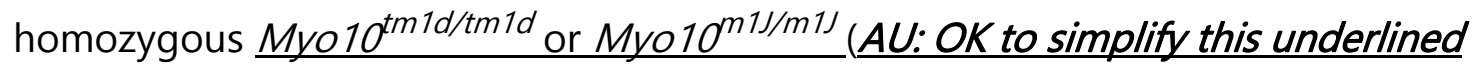

section just to $\mathrm{Myo}^{-/-}$?) null mutant mouse embryos die due to exencephaly, but a significant number still survive ${ }^{48,49}$. The brains of the surviving $M y 01 \sigma^{\prime-}$ embryos do not exhibit any gross abnormalities, but it remains to be seen if these animals have defects in cognitive function ${ }^{48,49}$. The phenotypes of the surviving $\mathrm{MyO}^{-\sigma^{-}}$mice are consistent with the loss of filopodia, including failed migration of melanocytes resulting in the appearance of a characteristic white belly spot, syndactyly (webbed digits), and abnormalities in the retinal vasculature due to persistent hyaloid vasculature ${ }^{48,49}$. The surprising survival of the $\mathrm{MyO}^{-\sigma^{-}}$mice and the $\sim 50 \%$ reduction in filopodia that occurs during retinal angiogenesis in these mutants indicates that cells and tissues have strategies to bypass the loss of MYO10 and can use alternative pathways to make filopodia.

\section{MYO10 and cancers}

Strong upregulation of MYO10 expression is associated with metastatic breast, skin and lung cancers ${ }^{50-53}$. Reduction of MYO10 expression in various metastatic cell lines significantly reduces filopodia production and diminishes invasion in 3D assays. Consistent with the important role of MYO10-induced filopodia in metastasis, induction 
of malignant melanoma in $\mathrm{MyO}^{-\alpha}$ mice results in smaller tumor size, reduced spread of cancer to the lungs and increased survival compared with that seen following malignant melanoma induction in wild-type mice $(A \cup: O K ?)^{4}$. Cancer cells can migrate through the ECM either individually or as part of a cell collective composed of a small population of leader cells at the front and followers that make up the main mass of this group of cells. Epigenetic changes modify the Myo10 genomic locus only in leader cells, resulting in increased MYO10 protein levels and robust production of filopodia ${ }^{53}$. These cells also secrete high levels of fibronectin compared with the followers, and as the cells move through the ECM they pull and align the fibronectin fibers. This action results in the formation of linear tracks that facilitate more efficient migration of the collective. Filopodia are stabilized by their interaction with fibronectin as the front of the leader cells advances and adheres strongly to the ECM. Interestingly, increasing filopodia number in leader cells by overexpression of MYO10 might be predicted to impair migration of the collective by generating more adhesion. However, increased numbers of filopodia actually enhance migration of the cell collective, which is likely due to increased alignment of the fibronectin matrix.

\section{Tunneling nanotubes and cytonemes}

Long filopodia-like extensions play critical roles in intercellular communication by serving as conduits for the transfer of signals from one cell to another. Tunneling nanotubes (TNTs) and cytonemes are slender (70-200 nm diameter) protrusions suspended between cells (i.e. they are not in contact with the substrate) that can reach lengths of $100 \mu \mathrm{m}$ (TNTs) to $300 \mu \mathrm{m}$ (cytonemes) ${ }^{55,56}$. These hollow tubes contain actin filaments, membrane vesicles and organelles, and even pathogens are actively transported within them. Cytonemes are critical for the long-distance delivery of morphogens such as Sonic hedgehog $(\mathrm{SHH})$ to differentiating tissues ${ }^{56}$. TNTs are observed both in cultured cells and tissues where they are typically open-ended the 
contact site for each cell. TNTs have been implicated in cancer chemoresistance and are used for the spread of pathogens and viruses ${ }^{55}$. For example, the HIV-1 accessory protein Nef stimulates MYO10-dependent TNT formation, promoting the cell-to-cell spread of the virus ${ }^{57}$. The mechanism of TNT formation is poorly understood, although it is thought that they might arise from dorsal filopodia, which require MYO10 for their formation. Overexpression of this myosin has been observed to increase both the number of these protrusions and vesicle transfer between cell partners, but whether MYO10 plays a transport role is currently unclear ${ }^{58}$. In contrast, recent work in cultured mouse cells strongly implicates MYO10 as the motor that transports morphogencontaining vesicles along the length of cytonemes to their target cell ${ }^{59}$. The MF domain of MYO10 is essential for its activity, indicating that it is bound to an adaptor protein present on $\mathrm{SHH}$-containing vesicles. $\mathrm{SHH}$ signaling is critical for neuronal development and its activity is notably diminished in the neural tube of $\mathrm{E} 9.5$ brains of $\mathrm{MyO}^{-1}$ mice. Interestingly, $\mathrm{SHH}$ signaling is not completely lost in these mice, indicating that a redundant or alternative pathway can promote some activation of $\mathrm{SHH}$ targets in the absence of cytonemes ${ }^{59}$. The role of MYO10 or related myosins in building both TNTs and cytonemes remains unclear at present.

\section{Future directions for filopodia and myosins}

There remain many open questions about how MYO10 contributes to filopodia formation and activity. Its mechanism of action during filopodia initiation is still undefined. In addition to acting as an actin organizer or a transporter, it might also serve to anchor membrane receptors such as integrins at the filopodia tip. The exact roles MYO10 plays and which factors control the mode of motor functionality is an interesting area for future study. Finally, MYO10 plays such an important role in filopodia formation in vertebrates that it is surprising to find that it has been lost in insects and nematodes (AU:OK?). These lineages have Myo7 and Myo15 and insects also 
have Myo22, which bears two MF domains in its tail region ${ }^{3}$. Perhaps one of these myosins could play a similar role in filopodia formation as DdMyo7, as suggested by recent work showing that fly Myo7a can bundle actin filaments when dimerized ${ }^{60}$. One of these myosins could also play a role in cytoneme formation. Alternatively, organisms could employ a purely actin-based mechanism for filopodia formation. Thus, the designation 'filopodia' likely defines a diverse group of thin cellular protrusions that may or may not require a myosin for formation.

\section{Myosins and microvilli}

The apical surface of epithelial cells that line the lumen of absorptive gut or kidney tubules is covered with a tightly packed carpet of microvilli that make up a brush border (Figure 1E-H). These projections are supported by a crosslinked core of 20-30 tightly bundled actin filaments with their plus ends at the tip and minus ends at the end of long rootlets embedded in a dense terminal web. Microvilli are strikingly uniform in length and held together at their tips by cadherin-based linkages.

\section{Microvillar myosins involved in membrane tension}

MY01A was the first mammalian unconventional myosin discovered. It is a $\mathrm{Ca}^{2+}$ regulated motor with a tail domain that directs binding to acidic lipids such as $\mathrm{PIP}_{2}{ }^{61}$, essential for crosslinking the actin core of microvilli to the overlying plasma membrane ${ }^{62}$ (Figure 4). Elegant studies established that MYO1A powers the flow of membranes from the base to the plus-end tips of microvilli and promotes the release of vesicles from the microvillar tips into the intestinal lumen ${ }^{63-65}$. These vesicles are enriched in alkaline phosphatase (which likely serves an antibacterial function by degrading bacterial lipopolysaccharide) and in key microvillar enzymes such as sucrase isomaltase. Whether this slow (30-60 $\mathrm{nm} \mathrm{s}^{-1}$ ), non-processive monomeric motor is assisted by a partner protein to carry out its functions remains unclear as its high abundance possibly suffices 
to maintain membrane tension. Surprisingly, in spite of the morphological abnormalities in the microvilli and the disorganization of the whole brush border in Myo1A null mutant mice, these mice do not exhibit any overt intestinal dysfunction ${ }^{65}$. However, this is likely due to compensation by increased levels of other myosins, such as MYO1C, in the microvillar core ${ }^{65}$. Loss of MYO1A results in colorectal cancer, suggestive of a role as a tumor suppressor, supported by the finding that it is critical for polarity maintenance in intestinal epithelial cells $(A U: O K)^{66}$.

Interestingly, loss of MYO6 localization from the intermicrovillar region has been reported in the Myo1A null mutant ${ }^{65}$. MYO6 is a tension-sensitive dimeric motor that uniquely moves towards the minus ends of actin filaments. It serves to transport receptors and channels to the base of microvilli where they undergo internalization via clathrin-mediated endocytosis. MYO6 thus controls the steady-state levels of proteins such as cystic fibrosis transmembrane conductance regulator (CFTR) and $\mathrm{Na}^{+} / \mathrm{H}^{+}$ exchanger 3 (NHE3) in the brush border, and loss of this motor protein correlates with increased levels of the CFTR channel on the microvillar membrane ${ }^{67}$. MYO6 also has an important role in maintaining the integrity of the brush border. Snell's waltzer ( $s v / s v)$ mice lacking MYO6 (AU:OK?) have an irregular brush border, where the membrane is no longer pulled down to the very base of the microvillar projections and fusion of microvilli is observed ${ }^{68}$. Interestingly, $M y o 1 A^{-1-} ; s v / s v$ double mutants do not exhibit any membrane tethering defects and the overall morphology and hexagonal packing of microvilli appears normal ${ }^{69}$. This restored morphology is associated with the appearance of MY01C, MYO1D, MY01E and MYO5A in the brush border, where they most likely compensate for the lack of the other myosins. However, it should be noted that the endocytic defects seen in the $s v / s v$ mutant are not rescued in the double mutants (AU:OK?). Thus, the action of MYO6, exerting a downward force on the microvillar membrane, opposes that of MYO1A, which generates membrane flow towards the tip 
(AU:OK?) providing a precise balance of forces to maintain the integrity of the microvillar membrane.

\section{MYO7B-dependent intermicrovillar links}

Microvilli are linked together at their tips by a heterophilic complex of the cadherins $\mathrm{CDHR} 2$ and $\mathrm{CDHR}^{70}$ (Figure 4), with small clusters of microvilli linked together at their tips bring observed during epithelial development. As the number of microvilli protruding from the apical surface increases, these protrusions all become linked at their tips, forming a network across the top of the cell ${ }^{70}$. The result is that these connections organize the microvilli into a tight, mechanically integrated network. The cytoplasmic tails of cadherins at the tips of microvilli are anchored to the actin core by the intermicrovillar adhesion complex (IMAC). This complex is composed of MYO7B, an MF myosin, the PDZ domain adaptor protein Harmonin-1 (USH1C) and the adaptor protein ANKS4B - a protein complex that is strikingly similar to the MYO7A-based USH1 complex found in stereocilia (see below). Microvilli of epithelial cells depleted for MYO7B lack the tip localization of IMAC components and of CDHR2 (AU: OK to say "and of CDHR2" here seeing as it is not strictly a component of the IMAC?), and the intermicrovillar links are visible along the microvillar length instead of just at the tips ${ }^{71}$. Similarly, loss of one of the two MYO7B light chains, CALML4, results in the loss of IMAC tip targeting, establishing the critical role of this subunit in MYO7B motor functionality ${ }^{72}$.

The IMAC is anchored to the microvillar actin core via the motor domain of MYO7B, providing a mechanism for adjusting tension across the links ${ }^{70}$. Loss of any one IMAC component causes loss of targeting of the other components to the tips and thus loss of the microvillar tip linkages ${ }^{70,73}$. A network of interactions connects the different members of the IMAC, with the PDZ adaptor protein USH1C playing a key role. Highresolution structures of the ANKS4B central intrinsically disordered region (CEN) bound 
to the MYO7B MF1 domain and the USH1C PDZ3 domain bound to the MYO7B MF2 domain provide insight into how the adaptor-motor IMAC complex is assembled ${ }^{70,74-76}$. In fact, several distinct complexes are possible, given the number of interaction sites between the different players ${ }^{76}$. Consistent with the multivalent interactions within the IMAC, a 1:1:1 mixture of USH1C, ANKS4B and the MYO7B MF domains can form a condensate via liquid-liquid phase separation both in vitro and when ectopically expressed in HeLa cells ${ }^{77}$. A cap of electron-dense material detected at the tips of microvilli could correspond to an IMAC condensate, providing a high local concentration of connections between cadherin and the actin cytoskeleton. Such a robust anchorage of the intermicrovillar cadherin links is critical for the brush border to withstand the mechanical stresses regularly experienced by the intestinal epithelium.

MYO7B has been implicated in mediating IMAC functions in two different ways: transport of the complex to the tip, and anchoring of the complex (AU:OK?) to actin to link microvillar tips to each other ${ }^{71,73}$. Movement of MYO7B towards the tip of a microvillus has been observed and loss of motor activity eliminates the formation of connections between microvillar tips, resulting in the disorganization of microvilli ${ }^{71}$. An in vivo 'pull-down' strategy showed that ANKS4B, USH1C and the cytoplasmic domains of either CDHR2 or CDHR5 can bind to the MYO7B tail independently, suggesting that MYO7B could transport these components individually to the $\mathrm{tip}^{78}$. The exact nature of the cargo that MYO7B transports to the microvillar tip - a subset of components, or all of them - is not known. MYO7B has relatively low ATPase activity but a high duty ratio, as is typical for motors that can act as tethers or anchors ${ }^{79}$. The clear localization of MYO7B to the microvillar tip also suggests that it must have dual roles as both a transporter and an anchor. Key questions then are what triggers the switch from a transporter to an anchor and does the MYO7B motor switch from operating as a dimer to functioning as a monomer? Such a switch might take place when the motor 
experiences a higher load, which likely requires the reorganization of the transported complex when it reaches the tip ${ }^{76}$. This could occur as a higher local concentration of IMAC components would favor integration into a dense network by liquid-liquid phase separation. How MYO7B motor activity affects the formation or stability of the condensate is currently undefined. Further studies are required to identify the factors that determine the precise position and geometry of the microvillar tip links during brush border maturation as well as the formation of the associated IMAC electron-dense regions.

\section{AU: add a subheading here? e.g. "Regulation of microvillar length"?}

The length of microvilli on a single cell is strikingly uniform, exhibiting only around $5 \%$ variation (AU:OK?) of the mean length $(\sim 1.1 \mu \mathrm{m})$ in the mouse small intestine, for example ${ }^{65}$. How is this length so precisely maintained? There appears to be a complex interplay between myosin-generated force, actin polymerization and intermicrovillar links that determines the consistent microvillar height across the brush border. Nonmuscle myosin IIC (MYH14, referred to here as MYO2C) is specifically enriched in brush border fractions where it is localized to the actin rootlets of microvilli, as well as at adherens junctions ${ }^{80,81}$. Surprisingly, inhibition of $\mathrm{MYO} 2 \mathrm{C}$ causes elongation of microvilli, disorganization of the brush border and a substantial reduction of the retrograde flow in the microvillar core in W4 cells, which are a useful model for early epithelial cell morphogenesis. Ectopic overexpression of $\mathrm{MYO} 2 \mathrm{C}$, on the other hand, results in a dramatic shortening of microvilli and mutations in the motor domain abrogate this phenotype ${ }^{80}$. These findings strongly implicate $\mathrm{MYO} 2 \mathrm{C}$ contractility, pulling on microvillar actin rootlets in the terminal web region. The effect of MYO2C on the retrograde flow of the actin bundle indicates that this motor counteracts the addition of actin monomers to the tip by promoting an optimal level of depolymerization at the rootlets (Figure 4). MYO7B might also play a role in controlling microvillar length. Cdhrz 
/- mice show a significant ( $50 \%)$ reduction in microvillar height and the loss of the CDHR2-based links distorts the shape of the microvilli; instead of the usual circular outline, the mutant microvilli are more oblong and the actin core appears irregular. The close packing of microvilli is also significantly disrupted ${ }^{82}$. Thus, transport of CDHR2 to the tip of microvilli may be crucial for dictating the coordinated length of adjacent protrusions. Co-expression of a chimeric motor comprising the MYO10 motor domain and the MYO7B tail with CDHR2 (AU:OK?) in HeLa cells robustly induces the formation of filopodia (AU: add something here like "a proxy for microvilli in these cells in culture"?). Interestingly, filopodia tips enriched in CDHR2 and the chimeric motor appear to be gathered together, with several of them being matched in height. Occasionally, one filopodia tip can be observed to slide along the side of another filopodium until it reaches that tip and stops growing ${ }^{78}$. While this heterologous system is obviously lacking the normal actin regulators that build microvilli, these intriguing findings implicate the tension generated from two tips being linked together as another potential player in precisely controlling and matching the height of adjacent microvilli.

\section{Myosins of the stereocilia}

The sensory cells of the auditory and vestibular epithelia in vertebrates are characterized by the presence of specialized apical projections known as hair bundles. Each contains 50-100 actin-filled stereocilia, arranged in ranks of successively increasing height, giving the bundle a staircase-like appearance (Figure 1I-L). The stereocilia are modified microvilli that can be up to $120 \mu \mathrm{m}$ in length ${ }^{83}$. The sensory hair cells of the inner ear have distinct functions - vestibular hair cells for balance, and cochlear inner and outer hair cells (IHCs and OHCs) for hearing by frequency detection - and distinct organizations in different parts of the inner ear (vestibular versus cochlear) ${ }^{84}$. During hair cell development, formation of the hair bundle involves establishment of planar polarity, building of stereocilia that are organized into their typical architecture, precise 
specification of stereocilia lengths, and formation of stereotypical links that join stereocilia together ${ }^{85,86}$. The acquisition of mechanotransduction and its influence on the development of the hair bundle are also important for stereocilia maturation ${ }^{87,88}$. Given the complexity of the system, this section will be confined to an overview of what is known about the function of myosins in $\mathrm{IHCs} \mathrm{s}^{10,85,88,89}$.

The hair bundle is a mechanotransducer composed of stereocilia that converts sound waves or orientation information into electrical signals ${ }^{90}$. Stereocilia are supported by a parallel bundle of actin filaments oriented with their minus ends in the rootlets that anchor them in the apical region of the hair cell. These protrusions are wider than microvilli, tapered at the bottom, and ordered in rows of graded height. The overall height of the bundle varies along the length of the sensory epithelium. Connections between stereocilia link them into an integrated bundle that acts as a single transduction unit. In the mouse cochlea, there are three rows of stereocilia (row 1 is the tallest) and the tops of the shorter stereocilia in rows 2 and 3 are linked to the side of the adjacent taller stereocilia via a cadherin-based structure known as the tip link (Figure 5). The cadherin PCDH15 makes up the lower half of the tip link, which is embedded in the top of the shorter stereocilia and is associated with the mechanotransduction channel (MET). The upper half of the tip link is composed of the cadherin $\mathrm{CDH} 23$; its membrane-spanning domain is embedded in the side of the taller, adjacent stereocilia. Sound waves cause a deflection of the bundle in the direction of the taller stereocilia, causing the bundle to pivot at its base (AU:OK?). The resulting strain transmitted via the tip link opens the MET channel, allowing ions to flow into the stereocilia ${ }^{90}$.

Genetic approaches have made substantial contributions to identifying roles for myosins in the hair cell, most notably uncovering the identity of genes mutated in Usher 
syndrome, the leading cause of combined deafness and blindness in the human population, as well as mouse deafness genes ${ }^{85}$. A combination of elegant biophysical, cell biological and single-cell proteomic studies has identified a diverse group of myosins required for stereocilia linkages, growth, length control and mechanotransduction (Figure 5).

\section{Myosins in adaptation and channel gating}

Opening of the MET channel causes depolarization of the cell followed by slow $\mathrm{Ca}^{2+}$ and actin-dependent adaptation. This resets tip-link tension and leads to MET channel closing, allowing it to operate at maximum sensitivity. A series of biophysical and chemical genetic studies revealed a role for MYO1C in slow adaptation ${ }^{91}$. MYO1C is a monomeric motor regulated by $\mathrm{Ca}^{2+}$ with a $\mathrm{PIP}_{2}$-binding domain in its tail and localizes to the upper tip link density. A related MYO1 family member, $\mathrm{MYO1H}$, is present in the hair bundle at similar levels as MYO1C and also appears to be localized to stereocilia tips $^{92}$. A small ensemble of MYO1C is thought to anchor the tip link to the stereocilia actin core by binding to the tail of $\mathrm{CDH} 23^{91}$. When tension is applied to the tip link, this non-processive motor slips down the actin core, reducing tension across the link and allowing the MET channel to close (i.e. adaptation). It is unknown, however, how $\mathrm{MYO} 1 \mathrm{H}$ contributes to this process, how MYO1C motors cluster when bound to $\mathrm{PIP}_{2}$, and whether other membrane-associated proteins are required for MYO1C organization or to connect these motors to the upper tip link (AU:OK?).

MYO7A is localized to the upper tip-link density and early work implicated this motor in MET channel transduction ${ }^{93,94}$. Hair bundles from Myo7a ${ }^{6 / 6 J}$ mutants exhibit abnormal mechano-electrical transduction, but, as they are mildly disorganized, it has been difficult to ascribe the defects solely to impaired MET gating ${ }^{94}$. Gene-edited mice that lack the canonical MYO7A isoform (Myo7a- $\Delta C$ ) have significantly reduced levels of 
MYO7A protein specifically in IHCs, with a loss of $60-70 \%$ of the protein from the upper tip-link density, yet hair bundle integrity is maintained (AU:OK?). The Myo7a- $\triangle C$ hair bundles exhibit a significant delay in the initial onset of current, consistent with the loss of a tension on the tip link ${ }^{95}$. The progressive deafness that results from the loss of the long (AU: could you clarify what you mean by long - canonical?) MYO7A isoform might be linked to a lower expression of MYO7A motors in these Myo7a- $\Delta C$ hair bundles. Alternatively, the 11 amino acid difference (AU: difference from what?) in the aminoterminal myosin sequence could result in drastically different motor properties under load, as has been seen for other myosins such as MYO1B and MYO14 ${ }^{96,97}$. These findings implicate MYO7A in tensioning the MET channel (AU:OK?) through anchoring the upper tip link to the actin core of the stereocilia and potentially providing a mechanically sensitive response to applied tension following hair bundle deflection. Further studies are needed to clarify how the anchoring properties of MYO7A are modulated by either $\mathrm{Ca}^{2+}$ signaling or tension (AU: "force" instead of "tension"?) to maintain and adjust tiplink tension.

\section{Myosins anchoring stereocilia links and the plasma membrane}

Top connectors, shaft connectors, lateral links and ankle links hold the hair bundle together ${ }^{85}$. Early studies revealed that these links are $\mathrm{Ca}^{2+}$ sensitive, implicating cadherin family members as key components ${ }^{98}$. The MF myosin MYO7A localizes along the length of the stereocilia and is concentrated where cadherin-based connectors link adjacent structures, at ankle links near the bottom of the stereocilia and at the upper tip-link density $^{93,99,100}$ (Figure 5). Mutations in the MYO7A gene in Usher syndrome type IB patients and studies of the Myo7A shaker-1 (sh $)$ mouse mutant establish this myosin as a central player in the formation and maintenance of a number of interstereociliary links ${ }^{101,102}$. The hair bundles from cochlear hair cells are notably disorganized and splayed in sh1 mice, similar to what is seen in the zebrafish Myo7a mariner mutant ${ }^{103,104}$. 
Characterization of Usher syndrome type I mutations led to the identification of an USH1 protein complex (AU:OK?) composed of USH1C (Harmonin), USH1G (SANS) and MYO7A that anchors the stereocilia cadherin links to the underlying actin core of the stereocilia, functioning in a similar way as the microvillar IMAC ${ }^{85}$. The multi-PDZ protein Harmonin is a central player in this complex: it can interact with the cytoplasmic tail of $\mathrm{CDH} 23$ at the upper tip-link density, with SANS and the MYO7A MF1 domain via canonical and non-canonical PDZ domain interactions, and with the MYO7A MF2 domain via its carboxy-terminal PDZ3 domain ${ }^{76,105,106}$. Interestingly, the short carboxyterminal extension of Harmonin PDZ3 also binds to its own PDZ1, an interaction that promotes an autoinhibited state that could serve to control interactions with MYO7A or SANS $^{76,107}$. As seen for the IMAC proteins, when ectopically expressed in HeLa cells the MYO7A tail, SANS and the Harmonin-1a isoform form a cytosolic liquid condensate upon liquid-liquid phase separation ${ }^{77}$. Interestingly, this condensate is abrogated by deafness-causing mutations in the MYO7A tail that are known to disrupt binding to the PDZ3 domain of Harmonin ${ }^{77}$, consistent with strong, multivalent interactions between these proteins playing a critical role in the formation of the dense condensate.

$\mathrm{CDH} 23$ is linked to MYO7A through Harmonin at the upper tip-link density. Disruption of the $\mathrm{CDH} 23$-binding site in Harmonin (AU:OK?) or the complete loss of all Harmonin isoforms disrupts the organization of cochlear hair cell bundles and alters the speed and extent of adaptation ${ }^{108,109}$, establishing the importance of Harmonin in the formation of stable interstereociliary links and mechanotransduction. A number of different Harmonin isoforms are expressed in the cochlear and vestibular sensory epithelia and have the potential to interact with a diverse set of binding partners ${ }^{110}$. While the interactions with SANS and the amino-terminal MYO7A MF1 domain are conserved in all Harmonin isoforms (AU:OK?) the potential for actin bundling or for 
binding to the MYO7A MF2 domain differs among isoforms. Thus, the varied interactions between these proteins might confer different mechanical properties on this network (AU:OK?) depending on the isoforms present ${ }^{75,76,99,106,111}$. A key question is what regulates the clustering of MYO7A motors via the formation of complexes in a Harmonin-isoform-dependent manner. Changes in the expression of individual Harmonin isoforms or in their levels are likely to play a role in dictating the type of MYO7A complex formed, but the presence of other binding partners could also be an important determinant (see below). Comprehensive cataloging of isoforms in hair bundles throughout development by single-cell proteomics and transcriptomics will undoubtedly provide answers to some of these questions in the near future $92,112,113$.

An outstanding question is whether MYO7A serves as a transporter, bringing USH1 (AU:OK?) complex members to various sites of action. MYO7A is a monomer which is regulated by head-tail autoinhibition, with the carboxy-terminal FERM domain inhibiting activity by binding to the motor domain ${ }^{114}$. MYO7A alone does not translocate along filopodia in HeLa cells but, when co-expressed with MyRIP (myosinVIla- and Rab-interacting protein), both move to the tips of filopodia ${ }^{115}$. This finding suggests that cargo binding both relieves autoinhibition and dimerizes (or oligomerizes) the motor allowing it to translocate along parallel actin filaments. One can envision that binding of Harmonin-SANS to the tail could also promote MYO7A dimerization to serve a similar function in stereocilia (AU:OK?).

MYO7A has been found to bind to other transmembrane adhesion linkers. Usher syndrome type II (USH2) is characterized by moderate hearing loss and retinitis pigmentosa. It results from mutations in two distinct transmembrane adhesion linkers, ADGVR1 (Vlgr1/GRP98; USH2C) and Usherin (USH2A), which are components of the ankle link ${ }^{116}$. Cochlear hair cells of mice lacking ADGVR1 exhibit significant 
disorganization ${ }^{116}$. MYO7A interacts with the cytoplasmic domains of Usherin and ADGVR1 via its MF2 domain. Both of these linkers are lost from ankle links in Myo7a mouse mutants, illustrating the central role that this motor has in either establishing and/or maintaining these connections ${ }^{116,117}$. Similar to what is seen with the USH1 complex, the cytoplasmic tails of the USH2 proteins interact with the PDZ domain cytosolic adaptors WHRN (whirlin, USH2D) and PDZD7, which also bind to the MYO7A tail $^{116-118}$. Mutations in WHRN and PDZD7 are associated with deafness ${ }^{119,120}$. Additionally, the transmembrane protein Vezatin is present in ankle links, where it interacts both with the cytoplasmic tail of Usherin and the MYO7A MF2 domain ${ }^{116,121}$. Thus, MYO7A is a core component of multiple distinct complexes critical for the formation and maintenance of ankle links at the base of stereocilia that are integral for the cohesion of the hair bundle. The diversity of interactions suggests a sophisticated mechanism for the timely formation and targeting of each complex throughout the development and lifespan of sensory hair cells.

Separation of individual stereocilia is maintained by tethering the apical membrane to the actin-dense cuticular plate that anchors the actin rootlets. MYO6 is found both in the cuticular plate and at the tapered base of the stereocilia. In the $\mathrm{sV} / \mathrm{sV}$ Myo6 mouse mutant, bulging of the membrane between stereocilia and club-like fused stereocilia are observed ${ }^{122-124}$. Thus, MYO6 plays a pivotal role in anchoring the stereocilia membrane to the cuticular plate, similar to its role in tethering the membrane to the base of microvilli68. MYO6's role in anchoring the membrane might be due to its role in endocytosis at the base of stereocilia (and microvilli) and/or in stabilizing the basal links between adjacent stereocilia, as $s v / s v$ mutants show a redistribution of basal linker proteins from the base to the shaft of the stereocilia ${ }^{125-127}$.

\section{Myosins control stereocilia length}


The length of individual stereocilia is precisely regulated both within the hair bundle and throughout the sensory epithelium (for example, along the length of the cochlea). Myosin activity contributes to stereocilia elongation via the delivery of actin regulators to the growing tips or by controlling the activity of these regulators (Figure 5). In addition, several different myosins interact with the cytoplasmic domain of tip links and loss of these myosins or inhibition of MET channel activity impacts stereocilia elongation. Thus, the control of stereocilia length requires a delicate partnership between actin dynamics, myosin activity and mechanotransduction, revealing that function and development are intertwined.

MYO3A and MYO3B regulate stereocilia extension and associate with the actin bundlers espin-1 (ESPN-1) and espin-like (ESPNL), which accumulate at stereocilia tips $^{128}$. These are unusual hybrid myosins with an amino-terminal Ser/Thr kinase domain, a central (actin-binding) motor domain and a short carboxy-terminal tail region, distinguished by the presence (MYO3A) or absence (MYO3B) of an actin-binding domain (tail homology domain II, THDII). These myosins are regulated by autophosphorylation, with phosphorylation of the motor significantly decreasing its affinity for actin and reducing motor activity ${ }^{128,129}$. MYO3A and MYO3B are monomeric; however, when a kinase-dead form of MYO3A is expressed in HeLa cells, it can move slowly along the length of filopodia to the tip, slowing filopodia dynamics and enhancing filopodia lifetime ${ }^{130,131}$. Mutations in the THDII actin-binding domain abolish this activity, revealing that tail binding allows MYO3A to move along the actin filament in an 'inchworm'-type fashion ${ }^{131,132}$. Co-expression of MYO3A and ESPN-1 in HeLa cells promotes filopodia elongation ${ }^{131}$. In contrast, MYO3B alone does not localize to filopodia tips in COS7 cells, consistent with the absence of the THDII domain. However, when co-expressed with ESPN-1 the two proteins are found at the tips and increased filopodia extension occurs ${ }^{133}$. Mutation of the actin-binding motif in ESPN-1 abolishes 
the tip localization of MYO3B, revealing that ESPN-1 provides a second actin-binding site that allows MYO3B to move along actin filaments. The key role of the motors when binding ESPN-1 is not only transporting this actin regulator but also activating it at the tip of stereocilia (AU:OK?). The shared (AU: common?) THDI tail region of these myosins binds to the ankyrin repeat region of ESPN-1, relieving their autoinhibition and enabling bundling of actin filaments ${ }^{134}$.

MYO3A, MYO3B and ESPN-1 localize to the tips of stereocilia in a thimble-like (AU: please clarify what you mean by thimble-like) distribution, while ESPNL is found only at the tips of rows 2 and 3 (the shorter stereocilia) 133,135,136. Loss of MYO3A results in late onset deafness in human patients (DFNB30) and in mice ${ }^{137-139}$, while MYO3B and ESPN-1 mutants do not exhibit any loss of hearing, even though the slope of the hair bundle staircase is altered in a subset of vestibular cells in the ESPN-1 nulls ${ }^{135}$. In the case of the ESPN-1 mutant, ESPNL likely compensates for the loss of ESPN-1 activity (AU:OK?). MYO3A is not essential for stereocilia formation, but its loss results in stereocilia degeneration after several months ${ }^{139}$ : the stereocilia are misshapen and longer than controls, revealing that MYO3A has a role in setting the final length of these protrusions $^{135,137,139}$. In contrast, a subtle decrease in the length of the shorter (row 2 and 3) stereocilia is observed in the $\mathrm{Myo3}^{-/-}$mutant. $M y o 3 A^{-/-}$Myo3 $B^{-1-}$ double mutant mice are profoundly deaf and exhibit global dysregulation in stereocilia organization and length with a loss of the graded height typically seen in hair bundles ${ }^{137}$. Thus, MYO3A and MYO3B are not required for stereocilia formation, but instead have shared roles in fine-tuning actin dynamics for setting or maintaining the proper graded length of stereocilia that is required for their function.

\section{Myosin control over the graded height of stereocilia}


Tremendous progress has been made in understanding how the lengths of the stereocilia in each row are specified and in identifying the key players required for elongation and maintenance of the distinct rows. Loss of the MF myosin MYO15A (Figure 2) in the shaker-2 (sh2) mouse mutant results in strikingly short stereocilia of uniform height in both cochlear and vestibular sensory hair cells, implicating this myosin as a key player in elongation and maintenance of stereocilia length ${ }^{140,141}$. The $s h 2$ mutation (C1779Y) likely disrupts nucleotide binding at the P-loop of MYO15A, establishing that its motor activity is essential for function ${ }^{141}$. Dimerized MYO15A moves at $300 \mathrm{~nm} \mathrm{~s}^{-1}$ and kinetic analysis demonstrates that MYO15A has a moderate duty ratio ${ }^{142,143}$. Ectopically expressed MYO15A moves towards the tips of filopodia in COS7 cells, consistent with a transporter role in stereocilia ${ }^{142,144}$. The ability to translocate to filopodia tips suggests that this myosin can either dimerize or has a second actinbinding site in its tail, similar to MYO3A. There are two MYO15A isoforms (MYO15A-L and MYO15A-S) that differ by the presence or absence of a 1,208 amino acid aminoterminal extension predicted to be largely disordered (but possibly involved in binding to other proteins $)^{145}$. The expression of these two MYO15A isoforms is temporally and spatially regulated during development ${ }^{146}$. During the early stages of stereocilia maturation, both isoforms are uniformly localized to the tips; however, maturation of the hair bundle towards a graded height arrangement of stereocilia correlates with MYO15A-S being exclusively present at the tips of the tallest stereocilia in row 1 and MYO15A-L at the tips of the shorter rows (2 and 3) where the MET channel resides (Figure 5). The differential localization of the two isoforms implicates the amino-terminal region in regulating the targeting of MYO15A-L to the shorter rows by a mechanism that is as yet not clear.

Careful and systematic analysis of a cohort of mouse mutants with phenotypes similar to those of the sh2 mutant has revealed a critical role for MYO15A in specifying 
row 1 height and has identified the key players that regulate and maintain row 1 identity. IHCs lacking either EPS8 (a regulator of actin dynamics), WHRN, GPSM2 (a GoLoco protein) or GNAI3 (Gai3, the $\alpha$ subunit of a heterotrimeric G protein) have an identical phenotype - short, immature hair bundles with excess stereocilia ${ }^{146-150}$. These mutant IHCs are similar in appearance to immature hair cells (E18.5) that also have a shallow staircase morphology and excessive stereocilia. These five proteins form a complex that is mainly localized to row 1 during maturation. MYO15A binds to the carboxy-terminal PDZ domain of WHRN via a PDZ-binding motif (PBM) that resides at its very carboxyl terminus and its MF2 domain binds the phosphotyrosine-binding (PTB) domain of EPS8. MYO15A appears to have a critical role in transporting EPS8 and WHRN to the tips of stereocilia, as both are lost from the tips of row 1 stereocilia in the sh2 mutant, but MYO15A localization is not altered in the WHRN (WI) (AU:OK?) mutant ${ }^{144,148}$. MYO15A-L and WHRN are co-transported to the tips of filopodia in COS7 cells, and when EPS8 is also present filopodia extension is enhanced. This is consistent with a MYO15A-WHRN-EPS8 complex promoting the extension of parallel actin bundles ${ }^{144,148}$. Both WHRN and EPS8 are enriched at the tips of the tallest stereocilia, and the levels of MYO15A-S and WHRN are directly correlated ${ }^{146,147}$. A GSPM2-GNAI3 complex is required to stabilize the MYO15A-S-EPS8-WHRN complex at the tip of row 1 stereocilia. Loss of either GPSM2 or GNAI3 results in the loss of the row 1 identity and the height differential across the three rows, with MYO15A-L and Esp8 now localizing to the tips of all stereocilia rows ${ }^{149,150}$. The signaling events that result in selective recruitment and stabilization of GSPM2 and GNAI3 to row 1 and how they, in turn, stabilize the MYO15AS-EPS8-WHRN complex are not yet known. The selective localization of WHRN and EPS8 to row 1 stereocilia by MYO15A-S is unexpected, given that MYO15A-L and MY015A-S both have a carboxy-terminal PBM, suggesting that the interaction between the MYO15A isoforms and their cargoes is tightly controlled in a context-dependent manner. The amino-terminal extension and carboxy-terminal tail of MYO15A-L interact 
(AU:OK?) to prevent binding of certain cargoes (such as WHRN or EPS8); perhaps this autoinhibitory interaction specifically blocks the interaction of the PBM with WHRN ${ }^{146}$.

Recent work showed that the MF2-PBM binding region of MYO15 can form a dense condensate with WHRN and the amino-terminal PTB-WHRN-binding domain (AU:OK?) of EPS8 both in vitro and when all three proteins are ectopically expressed in cells, similar to what is seen for the MYO7A and MYO7B tails and their partners Harmonin and SANS/ANKS4B (Figure 2) 77,151 . The ability of EPS8 to bind actin and the presence of dense material at the tip of stereocilia suggests that a MYO15-mediated condensate could serve to anchor actin filaments to the membrane at the tip. Interestingly, the condensate does promote actin bundling in vitro with droplets seen to be decorating the filaments. Much remains to be learned about the interactions between MYO15, WHRN and EPS8 and their potential role in linking it (AU: the condensate?) to the stereocilia tip. As is the case with MYO7A and MYO7B, it remains to be seen how myosin motor activity may impact the assembly and transport of the complex as well as condensate formation.

\section{Role of mechanotransduction}

The development of the cochlear IHC hair bundles in mice involves both widening and lengthening of stereocilia, processes that occur in alternate phases ${ }^{152}$. The first lengthening phase occurs during embryonic development and is followed by widening, with row 2 increasing most notably (P0-P4.5). Then, a second phase of stereocilia lengthening occurs, with row 1 dramatically increasing its length to $6 \mu \mathrm{m}(\mathrm{P} 7.5-19.5)^{87}$. The widening phase for row 2 stereocilia correlates with the onset of mechanotransduction. Mutants lacking mechanotransduction due to loss of the transmembrane-like channels TMC1 and TMC2 or their targeting partner TMIE exhibit distinct alterations in hair bundle morphology. Notably, the staircase morphology is less 
significant (AU: 'disrupted' instead of 'less significant'?) and the widening of row 2 stereocilia that occurs concomitantly with the onset of mechanotransduction is no longer observed. While some differences are observed between the channel mutants, MYO15A-S, EPS8, WHRN and GNAI3 are all present at the tips of row 1 and row 2 stereocilia, with the shift in distribution becoming more pronounced as development proceeds from P7.5 to P21.5. This change appears specific for these row 1 proteins since the tip localization of MYO3A and ESPN-1 is unchanged. Loss of mechanotransduction also impacts the localization of MYO15A-L, which is then found at the tips of stereocilia in all rows. Interestingly, sh2 mutants show defects in stereocilia widening in IHCs (and $\mathrm{OHCs}$, resulting in stereocilia of equal diameter in all three rows, similar to what is seen in the transduction mutants ${ }^{153}$, while the normal changes in stereocilia widening or thinning are maintained in the $M y 015 A^{\Delta N / \Delta N}$ mutant, which specifically lacks the MY015A-L isoform. Thus, in addition to playing a key role in the graded height of stereocilia, MYO15A-S is critical for controlling the programmed changes in their width. The mechanism for how protein distributions change at the tip during the onset of mechanotransduction and for how changes to the specification of row 1 impact actin dynamics to promote increasing stereocilia thickness in row 2 during the onset of mechanotransduction are unclear at present $(A \cup: O K ?)^{87,153}$. Together, these findings establish that there is an exquisite balance between mechanotransduction and development of the mature hair bundle architecture that relies on MYO15A-Sdependent elongation of row 1.

\section{Summary and perspective}

The formation and function of filopodia, microvilli and stereocilia require a variety of myosin family members with distinct roles that coordinate their action with regulators of actin polymerization. The MF family of myosins play particularly prominent roles in promoting or regulating the growth of these structures (MYO10 - filopodia; MYO15 - 
stereocilia) and in anchoring adhesion receptors to their actin core (MYO10 - integrin; MYO7, MYO15 - cadherin family members). With their motor activity, these myosins power their translocation along these special actin-based structures, enabling them to deliver actin regulators to their tips, and transport channels, membrane or receptors along their length to the tip or the base. Many myosins are localized to specific regions of filopodia, microvilli and stereocilia where anchoring or adhesion receptors reside. There, they can act as tension-sensitive anchors that sense and respond to mechanical changes during extension or bundling of the protrusion. MYO1 and MYO6 also have roles in driving membrane flow or anchoring the membrane to the actin-rich apical surface (AU: cortex?) of epithelial cells, in addition to anchoring proteins to the actin core of the protrusion. Retrograde flow of the core actin filaments is important for regulating extension or generating tension along the length of filopodia and microvilli, thereby contributing to stabilization of the protrusion and regulation of its growth. The diverse motor properties of all of these myosins make each of them well-tuned to serve their function as either a transporter or an anchor in filopodia, microvilli and stereocilia. The exact role that each motor plays is also defined locally and temporally, depending on their association with specific partners.

Many fundamentally important questions remain about the function of myosins in filopodia, microvilli and stereocilia. There is a delicate balance between protrusion, formation and stabilization, although the chemical and mechanical signaling that controls the activity of motors and actin polymerization factors required for these processes is not fully defined. The timely formation of protrusions relies on the activities of closely related homologs or different isoforms of both the myosin motors and their adaptors, especially in the case of microvilli and stereocilia (for example, MYO15A-S and MYO15A-L). The spatiotemporal control of isoform expression and activity is poorly understood at present. For example, what determines the expression of specific proteins 
to define the different stereocilia rows and which factors determine the onset of elongation and widening phase are some of the most intriguing questions. There are also many gaps in our knowledge about the biochemical properties of the different isoforms and how these impact protrusion function and the composition of various complexes at tips and sites of adhesion. Thus, much remains to be learned about how motors build filopodia, microvilli and stereocilia and contribute to their function. Progress in the coming years will undoubtedly uncover new principles of motor operation and how motors and their tracks work together.

\section{Acknowledgements}

A.H. is supported by an IRP grant from CNRS, ANR-17-CE11-0029-01, ANR-19-CE110015-02. The A.H. team is part of the Labex Cell(n)Scale (ANR-11-LABX-0038), which is part of the IDEX PSL (ANR-10-IDEX-0001-02). M.A.T. is supported by the NIH National Institute of General Medical Sciences (R01GM122917).

Figure 1. Features of filopodia, microvilli and stereocilia.

(A) Platinum replica micrograph of a filopodium extending from the lamella of a BG2 fly neural cell, scale $=1 \mu \mathrm{m}$. (B) Filopodia emerging from the lamella of a BG2 cell, scale = $1 \mu \mathrm{m}$. (C) Dictyostelium amoeba expressing GFP-DdMyo7 stained for F-actin (red) (AU:OK?) scale $=5 \mu \mathrm{m}$. (D) Time course (in seconds) of a HeLa cell expressing EGFPMYO10 (top) and mCherry-actin (middle) extending a filopodium, showing punctae assembling on the membrane that give rise to a new filopodium, scale $=2 \mu \mathrm{m}$. (E) Scanning electron micrograph of the apical surface of differentiating human intestinal (AU:OK?) Caco2-BBE cells 2 days post confluency (DPC2) showing microvillar buds (yellow arrows) and longer microvilli clustered at their tips (arrowheads), scale $=500 \mathrm{~nm}$. 
(F) Scanning electron micrograph at DPC8 showing clustering of microvillar tips and the links between them (green circles), scale = each side is $50 \mu \mathrm{m}$. (AU: what does the 2 at top left correspond to?) (G) Structured illumination microscopy of Caco2BBE cells expressing EGFP-MYO7B (green) stained for F-actin (red). Distal tip enrichment is indicated by arrows, scale $=$ each side is $50 \mu \mathrm{m}$. (AU: what does the 2 at top left correspond to?) (H) Freeze-etch electron micrograph of the apical surface of the mouse small intestine (SI) showing the organization of linked microvilli, scale $=100 \mathrm{~nm}$. (I) Scanning electron micrograph of the auditory epithelium from a P8 mouse showing the organization of the outer and inner hair cells $(\mathrm{OHC}, \mathrm{IHC})$, scale $=1 \mu \mathrm{m}$. (J) Scanning electron micrograph of IHC stereocilia at P4, boxed region at the top is magnified below. Note the interstereocilia links. (K) IHC stained for MYO15-L (green - tips of row 2 and 3) and actin (red), scale $=500 \mathrm{~nm}$. (L) Stimulated emission depletion (STED) microscopy image showing actin (purple) and GPSM2 (green) localized to the tip of row 1 stereocilia, scale $=2 \mu \mathrm{m}$. $(A, B)$ Reproduced with permission from ${ }^{154}$ (C) The Company of Biologists. (C) Image from ${ }^{6}$. (D) Reproduced with permission from ${ }^{33}$ (CC BY 4.0). $(\mathrm{E}, \mathrm{H})$ Reproduced with permission from ${ }^{70}$. $(F, G)$ Images from ${ }^{71}$. (I) Reproduced with permission from ${ }^{155}$ (C) The Company of Biologists. (J,K) Images from ${ }^{146}$ (C) 2015, Nicolson (CC BY 4.0). (L) Image from ${ }^{149}$ (CC BY 4.0).

Figure 2. Myosin family members with roles in filopodia, microvilli and stereocilia. Schematics illustrating the key domains and features of diverse myosin motors. Key features are annotated and interactions between domains and partners or targets are indicated with arrows. Note that some myosins may use other light chains (LCs) in addition to calmodulin (CaM), including essential and regulatory LCs (ELC, RLC), calmodulin-related LC (CALM4, CLP). SAH, stable $\alpha$-helix; PEST, proline (P), glutamic acid ( $\mathrm{E})$, serine $(\mathrm{S})$, and threonine $(\mathrm{T})$ rich sequence; $\mathrm{PH}$, pleckstrin homology; MF, MyTH4FERM; PIP $(3,4,5) \mathrm{P}_{3}$, phosphatidylinositol $(3,4,5)$-trisphosphate; $\mathrm{MB}$, membrane binding; 
3HB, three-helix bundle; $C B D$, cargo-binding domain; $S / T$ kinase, serine/threonine kinase; 3THDI/II, tail homology domain I or II; SH3, Src homology 3; CT, cytoplasmic tail; Ank, Ankryin repeat region; CEN, central domain; SAM, sterile alpha motif; PBM, PDZbinding motif; Nter/Cter, amino terminus and carboxyl terminus; PDZ, post-synaptic density protein (PSD95), Drosophila disc large tumor suppressor (Dlg1), and zonula occludens-1 protein (ZO-1); WH2, WASP homology 2; ABM, actin-binding module; PTB, phosphotyrosine-binding domain; WBD, whirlin-binding domain; Pro, Proline-rich; HHD, harmonin homology domain.

Figure 3. Model of MYO10 function in filopodia formation and function.

Schematic illustration of the activation and role of MYO10 in filopodia initiation, transport of VASP towards the growing filopodia tip, and anchoring integrins at the filopodia tip. (A) MYO10 is a monomer prior to activation, and binding to $\mathrm{PI}(3,4,5) \mathrm{P}_{3}(\boldsymbol{A U}$ : I will ensure that the second 3 is subscript in a revised version of the figure! And I will also change Myo to MYO in the key.) opens up the molecule and promotes activation and dimerization (steps 1 and 2). (AU: please add a brief description of steps 3-6) Green arrows indicate filopodia extension as actin monomers are added to the tip of the growing filopodium. The blue arrow represents myosin-driven cargo transport. (B) MYO10 within the filopodium can translocate along the actin core or return to the cytosol via retrograde flow generated by MYO2A. (AU: include some brief details regarding integrins and talin and what is represented by the anchor?) Red arrows indicate the downward pulling force exerted by MYO2A filaments in the cortex. ECM, extracellular matrix (highlighted with grey circle).

Figure 4. The myosins of microvilli. 
Conceptual model of the diverse functions carried out by the different microvillar myosins. These include generation of membrane flow by MYO1A (light blue arrows) that leads to shedding of membrane vesicles containing alkaline phosphatase (AP). Green arrows indicate microvillar extension as monomers are added to the tip. MYO6 transports channels (such as NHE3 shown here) down to the base of the microvillus, and also keeps the basal plasma membrane between the microvilli tightly anchored to the actin cortex (grey arrows). MYO7B-Harmonin-ANKS4B may move components of the IMAC complex including Harmonin, ANKS4B, and the cadherins CDHR2 and CDHR5 up toward the microvillar tip (black arrows). There, an IMAC condensate that includes MYO7B could anchor the cadherins in place thus stabilizing the intermicrovillar links. Non-muscle MYO2C (MYH14 - AU: I will change NMY14 to MYH14 in the key) links actin rootlets and provides a downward pulling force (orange arrows) balanced by polymerization at the microvillar tip to maintain proper microvillar length.

Figure 5. Functions of stereocilia myosins.

Illustration of the roles of myosins in controlling the length of stereocilia, linking adjacent stereocilia, and anchoring the tip link at postnatal day 4.5 (left) and postnatal day 15 (right). MYO3A and MYO3B play a role in the transport of the actin-bundling protein espin and regulate the length of the growing stereocilia. MYO15A also acts as a transporter, carrying the adaptor WHRN and the actin regulator Eps8 to the tips of all stereocilia at P4.5 to promote stereocilia growth. (AU: apologies for only asking this now, but why are there two row 1s for P4.5?) The long isoform MYO15A-L (AU: do you mean the short isoform MYO15A-S?) is localized most notably in row 1 at P15 where it forms a complex with Eps8 and WHRN that binds to GPSM2-GNAl, stimulating actin polymerization activity and driving stereocilia elongation. In contrast, the short isoform MYO15A-S (AU: do you mean long isoform MYO15A-L?) is found only in rows 2 and 3 (AU: it is not shown in row 2 - should it be added? Also / shall get an L added to the 
MYO15A-L shown in row 3.), where it stabilizes these shorter stereocilia. Several MYO1C molecules are likely associated at the upper tip link density (AU: add here "between rows 1 and 2"?), clustered by an unknown mechanism (indicated by ?) where they contribute to slow adaptation. MYO7A is also present at the upper tip link density (AU: add "between rows 2 and 3"?) where it serves as the tip link motor, tensioning the MET channel. MYO7A also associates with ankle links that contribute to holding the hair bundle together. MYO6 is concentrated at the base of the stereocilia in the actin-dense rootlets where it anchors the membrane to the actin-rich cuticular plate (not shown). 


\section{References}

1. Cavalier-Smith, T., and Chao, E.E. (2003). Phylogeny and classification of phylum Cercozoa (Protozoa). Protist 154, 341-358.

2. Hanousková, P., Táborský, P., and Čepička, I. (2019). Dactylomonas gen. nov., a novel lineage of Heterolobosean flagellates with unique ultrastructure, closely related to the Amoeba Selenaion koniopes Park, De Jonckheere \& Simpson, 2012. J. Eukaryot. Microbiol. 66, 120-139.

3. Kollmar, M., and Muhlhausen, S. (2017). Myosin repertoire expansion coincides with eukaryotic diversification in the Mesoproterozoic era. BMC Evol. Biol. 17, 211.

4. Yabuki, A., Ishida, K., and Cavalier-Smith, T. (2013). Rigifila ramosa n. gen., n. sp., a filose apusozoan with a distinctive pellicle, is related to Micronuclearia. Protist $164,75-$ 88.

5. Parra-Acero, H., Harcet, M., Sánchez-Pons, N., Casacuberta, E., Brown, N.H., Dudin, O., and Ruiz-Trillo, I. (2020). Integrin-mediated dhesion in the unicellular holozoan Capsaspora owczarzaki. Curr. Biol. 30, 4270-4275.e4.

6. Tuxworth, R.I., Weber, I., Wessels, D., Addicks, G.C., Soll, D.R., Gerisch, G., and Titus, M.A. (2001). A role for myosin VII in dynamic cell adhesion. Curr. Biol. 11, 318-329.

7. Dayel, M.J., and King, N. (2014). Prey capture and phagocytosis in the choanoflagellate Salpingoeca rosetta. PLoS One 9, e95577.

8. Sebe-Pedros, A., Burkhardt, P., Sanchez-Pons, N., Fairclough, S.R., Lang, B.F., King, N., and Ruiz-Trillo, I. (2013). Insights into the origin of metazoan filopodia and microvilli. Mol. Biol. Evol. 30, 2013-2023.

9. Crawley, S.W., Mooseker, M.S., and Tyska, M.J. (2014). Shaping the intestinal brush border. J. Cell Biol. 207, 441-451.

10. Barr-Gillespie, P.G. (2015). Assembly of hair bundles, an amazing problem for cell biology. Mol. Biol. Cell. 26, 2727-2732.

11. Coluccio, L.M. (2020). Myosins (Cham, Switzerland: Springer). 
12. Cook, A.W., and Toseland, C.P. (2021). The roles of nuclear myosin in the DNA damage response. J. Biochem. https://doi.org/10.1093/jb/mvaa113.

13. Masters, T.A., Kendrick-Jones, J., and Buss, F. (2017). Myosins: domain organisation, motor properties, physiological roles and cellular functions. Handb. Exp. Pharmacol. 235, 77-122.

14. Robert-Paganin, J., Pylypenko, O., Kikuti, C., Sweeney, H.L., and Houdusse, A. (2020). Force generation by myosin motors: a structural perspective. Chem. Rev. 120, 535.

15. Trivedi, D.V., Nag, S., Spudich, A., Ruppel, K.M., and Spudich, J.A. (2020). The myosin family of mechanoenzymes: from mechanisms to therapeutic approaches. Annu. Rev. Biochem. 89, 667-693.

16. Weck, M.L., Grega-Larson, N.E., and Tyska, M.J. (2017). MyTH4-FERM myosins in the assembly and maintenance of actin-based protrusions. Curr. Opin. Cell Biol. 44, 6878.

17. Gallop, J.L. (2020). Filopodia and their links with membrane traffic and cell adhesion. Semin. Cell. Dev. Biol. 102, 81-89.

18. Jacquemet, G., Baghirov, H., Georgiadou, M., Sihto, H., Peuhu, E., Cettour-Janet, P., He, T., Perälä, M., Kronqvist, P., Joensuu, H., et al. (2016). L-type calcium channels regulate filopodia stability and cancer cell invasion downstream of integrin signalling. Nat. Commun. 7, 13297.

19. McClay, D.R. (1999). The role of thin filopodia in motility and morphogenesis. Exp. Cell Res. 253, 296-301.

20. Aliyu, I.A., Kumurya, A.S., Bala, J.A., Yahaya, H., and Saidu, H. (2020). Proteomes, kinases and signalling pathways in virus-induced filopodia, as potential antiviral therapeutics targets. Rev. Med. Virol. eRMV2202.

21. Heckman, C.A., and Plummer, H.K. (2013). Filopodia as sensors. Cell. Signal. 25, 2298-2311.

22. Jacquemet, G., Stubb, A., Saup, R., Miihkinen, M., Kremneva, E., Hamidi, H., and Ivaska, J. (2019). Filopodome mapping identifies p130Cas as a mechanosensitive regulator of filopodia stability. Curr. Biol. 29, 202-216.e7. 
23. Yang, C., and Svitkina, T. (2011). Filopodia initiation: focus on the Arp2/3 complex and formins. Cell Adh. Migr. 5, 402-408.

24. Mogilner, A., and Rubinstein, B. (2005). The physics of filopodial protrusion. Biophys. J. 89, 782-795.

25. Bohil, A.B., Robertson, B.W., and Cheney, R.E. (2006). Myosin-X is a molecular motor that functions in filopodia formation. Proc. Natl. Acad. Sci. USA 103, 12411-12416.

26. Lu, Q., Ye, F., Wei, Z., Wen, Z., and Zhang, M. (2012). Antiparallel coiled-coilmediated dimerization of myosin X. Proc. Natl. Acad. Sci. USA 109, 17388-17393.

27. Ropars, V., Yang, Z., Isabet, T., Blanc, F., Zhou, K., Lin, T., Liu, X., Hissier, P., Samazan, F., Amigues, B., et al. (2016). The myosin $X$ motor is optimized for movement on actin bundles. Nat. Commun. 7, 12456.

28. Vavra, K.C., Xia, Y., and Rock, R.S. (2016). Competition between coiled-coil structures and the impact on Myosin-10 bundle selection. Biophys. J. 110, 2517-2527.

29. Arthur, A.L., Songster, L.D., Sirkia, H., Bhattacharya, A., Kikuti, C., Borrega, F.P., Houdusse, A., and Titus, M.A. (2019). Optimized filopodia formation requires myosin tail domain cooperation. Proc. Natl. Acad. Sci. USA 116, 22196-22204.

30. Petersen, K.J., Goodson, H.V., Arthur, A.L., Luxton, G.W., Houdusse, A., and Titus, M.A. (2016). MyTH4-FERM myosins have an ancient and conserved role in filopod formation. Proc. Natl. Acad. Sci. USA 113, E8059-E8068.

31. Tokuo, H., Mabuchi, K., and Ikebe, M. (2007). The motor activity of myosin-X promotes actin fiber convergence at the cell periphery to initiate filopodia formation. J. Cell Biol. 179, 229-238.

32. Masters, T.A., and Buss, F. (2017). Filopodia formation and endosome clustering induced by mutant plus-end-directed myosin VI. Proc. Natl. Acad. Sci. USA 114, 15951600.

33. He, K., Sakai, T., Tsukasaki, Y., Watanabe, T.M., and Ikebe, M. (2017). Myosin X is recruited to nascent focal adhesions at the leading edge and induces multi-cycle filopodial elongation. Sci. Rep. 7, 13685. 
34. Ikebe, M., Sato, O., and Sakai, T. (2018). Myosin X and cytoskeletal reorganization. Appl. Microsc. 48, 33-42.

35. Arthur, A.L., Crawford, A., Houdusse, A., and Titus, M.A. (2021). VASP-mediated actin dynamics recruit and activate filopodia myosin. bioRxiv, https://doi.org/10.1101/2021.03.16.435667.

36. Umeki, N., Jung, H.S., Sakai, T., Sato, O., Ikebe, R., and Ikebe, M. (2011). Phospholipid-dependent regulation of the motor activity of myosin X. Nat. Struct. Mol. Biol. 18, 783-788.

37. Plantard, L., Arjonen, A., Lock, J.G., Nurani, G., Ivaska, J., and Stromblad, S. (2010). PtdIns $(3,4,5) \mathrm{P}_{3}$ is a regulator of myosin-X localization and filopodia formation. J. Cell Sci. $123,3525-3534$.

38. Tokuo, H., and Ikebe, M. (2004). Myosin X transports Mena/VASP to the tip of filopodia. Biochem. Biophys. Res. Commun. 319, 214-220.

39. Lin, W.H., Hurley, J.T., Raines, A.N., Cheney, R.E., and Webb, D.J. (2013). Myosin X and its motorless isoform differentially modulate dendritic spine development by regulating trafficking and retention of vasodilator-stimulated phosphoprotein. J. Cell Sci. $126,4756-4768$.

40. Nagy, S., Ricca, B.L., Norstrom, M.F., Courson, D.S., Brawley, C.M., Smithback, P.A., and Rock, R.S. (2008). A myosin motor that selects bundled actin for motility. Proc. Natl. Acad. Sci. USA 105, 9616-9620.

41. Kerber, M.L., Jacobs, D.T., Campagnola, L., Dunn, B.D., Yin, T., Sousa, A.D., Quintero, O.A., and Cheney, R.E. (2009). A novel form of motility in filopodia revealed by imaging Myosin-X at the single-molecule level. Curr. Biol. 19, 967-973.

42. Brawley, C.M., and Rock, R.S. (2009). Unconventional myosin traffic in cells reveals a selective actin cytoskeleton. Proc. Natl. Acad. Sci. USA 106, 9685-9690.

43. Alieva, N.O., Efremov, A.K., Hu, S., Oh, D., Chen, Z., Natarajan, M., Ong, H.T., Jégou, A., Romet-Lemonne, G., Groves, J.T., et al. (2019). Myosin IIA and formin dependent mechanosensitivity of filopodia adhesion. Nat. Commun. 10, 3593.

44. Almagro, S., Durmort, C., Chervin-Petinot, A., Heyraud, S., Dubois, M., Lambert, O., Maillefaud, C., Hewat, E., Schaal, J.P., Huber, P., et al. (2010). The motor protein Myosin-X 
transports VE-cadherin along filopodia to allow the formation of early endothelial cellcell contacts. Mol. Cell Biol. 30, 1703-1717.

45. Zhang, H., Berg, J.S., Li, Z., Wang, Y., Lang, P., Sousa, A.D., Bhaskar, A., Cheney, R.E., and Stromblad, S. (2004). Myosin-X provides a motor-based link between integrins and the cytoskeleton. Nat. Cell Biol. 6, 523-531.

46. Miihkinen, M., Grönloh, M.L.B., Vihinen, H., Jokitalo, E., Goult, B.T., Ivaska, J., and Jacquemet, G. (2020). Myosin-X FERM domain modulates integrin activity at filopodia tips. bioRxiv, https://doi.org/10.1101/2020.05.05.078733.

47. Efremov, A.K., Yao, M., Sheetz, M.P., Bershadsky, A.D., Martinac, B., and Yan, J. (2020). Mechanosensitive calcium signaling in filopodia. bioRxiv, https://doi.org/10.1101/2020.10.21.346247.

48. Bachg, A.C., Horsthemke, M., Skryabin, B.V., Klasen, T., Nagelmann, N., Faber, C., Woodham, E., Machesky, L.M., Bachg, S., Stange, R., et al. (2019). Phenotypic analysis of Myo10 knockout (Myo10 $\mathrm{m}^{\mathrm{tm} / \mathrm{tm} 2}$ ) mice lacking full-length (motorized) but not brainspecific headless myosin X. Sci. Rep. 9, 597.

49. Heimsath, E.G., Yim, Y.I., Mustapha, M., Hammer, J.A., and Cheney, R.E. (2017). Myosin-X knockout is semi-lethal and demonstrates that myosin-X functions in neural tube closure, pigmentation, hyaloid vasculature regression, and filopodia formation. Sci. Rep. 7, 17354.

50. Arjonen, A., Kaukonen, R., Mattila, E., Rouhi, P., Högnäs, G., Sihto, H., Miller, B.W., Morton, J.P., Bucher, E., Taimen, P., et al. (2014). Mutant p53-associated myosin-X upregulation promotes breast cancer invasion and metastasis. J. Clin. Invest. 124, 10691082.

51. Cao, R., Chen, J., Zhang, X., Zhai, Y., Qing, X., Xing, W., Zhang, L., Malik, Y.S., Yu, H., $Z$ Zhu, $X$. , et al. (2014). Elevated expression of myosin $X$ in tumours contributes to breast cancer aggressiveness and metastasis. Br. J. Cancer. 111, 539-550.

52. Shibue, T., Brooks, M.W., Inan, M.F., Reinhardt, F., and Weinberg, R.A. (2012). The outgrowth of micrometastases is enabled by the formation of filopodium-like protrusions. Cancer Discov. 2, 706-721.

53. Summerbell, E.R., Mouw, J.K., Bell, J.S.K., Knippler, C.M., Pedro, B., Arnst, J.L., Khatib, T.O., Commander, R., Barwick, B.G., Konen, J., et al. (2020). Epigenetically 
heterogeneous tumor cells direct collective invasion through filopodia-driven fibronectin micropatterning. Sci. Adv. 6, eaaz6197.

54. Tokuo, H., Bhawan, J., and Coluccio, L.M. (2018). Myosin X is required for efficient melanoblast migration and melanoma initiation and metastasis. Sci. Rep. 8, 10449.

55. Cordero Cervantes, D., and Zurzolo, C. (2021). Peering into tunneling nanotubesThe path forward. EMBO J., e105789.

56. Kornberg, T.B. (2014). Cytonemes and the dispersion of morphogens. Wiley Interdiscip. Rev. Dev. Biol. 3, 445-463.

57. Uhl, J., Gujarathi, S., Waheed, A.A., Gordon, A., Freed, E.O., and Gousset, K. (2019). Myosin- $X$ is essential to the intercellular spread of HIV-1 Nef through tunneling nanotubes. J. Cell Commun. Signal. 13, 209-224.

58. Gousset, K., Marzo, L., Commere, P.H., and Zurzolo, C. (2013). Myo10 is a key regulator of TNT formation in neuronal cells. J. Cell Sci. 126, 4424-4435.

59. Hall, E.T., Dillard, M.E., Stewart, D.P., Zhang, Y., Wagner, B., Levine, R.M., PruettMiller, S.M., Sykes, A., Temirov, J., Cheney, R.E., et al. (2021). Cytoneme delivery of Sonic Hedgehog from ligand-producing cells requires Myosin 10 and a Dispatched-

BOC/CDON co-receptor complex. elife 10, e61432.

60. Liu, R., Billington, N., Yang, Y., Bond, C., Hong, A., Siththanandan, V., Takagi, Y., and Sellers, J.R. (2021). A binding protein regulates myosin-7a dimerization and actin bundle assembly. Nat. Commun. 12, 563.

61. Coluccio, L.M. (2008). Myosin I. In Myosins: A Superfamily of Molecular Motors, L.M. Coluccio, ed. (Dordrecht, The Netherlands: Springer), pp. 95-124.

62. Nambiar, R., McConnell, R.E., and Tyska, M.J. (2009). Control of cell membrane tension by myosin-I. Proc. Natl. Acad. Sci. USA 106, 11972-11977.

63. McConnell, R.E., and Tyska, M.J. (2007). Myosin-1a powers the sliding of apical membrane along microvillar actin bundles. J. Cell Biol. 177, 671-681.

64. McConnell, R.E., Higginbotham, J.N., Shifrin, D.A., Tabb, D.L., Coffey, R.J., and Tyska, M.J. (2009). The enterocyte microvillus is a vesicle-generating organelle. J. Cell Biol. 185, 1285-1298. 
65. Tyska, M.J., Mackey, A.T., Huang, J.D., Copeland, N.G., Jenkins, N.A., and Mooseker, M.S. (2005). Myosin-1a is critical for normal brush border structure and composition. Mol. Biol. Cell 16, 2443-2457.

66. Mazzolini, R., Dopeso, H., Mateo-Lozano, S., Chang, W., Rodrigues, P., Bazzocco, S., Alazzouzi, H., Landolfi, S., Hernandez-Losa, J., Andretta, E., et al. (2012). Brush border Myosin la has tumor suppressor activity in the intestine. Proc. Natl. Acad. Sci. USA 109, 1530-1535.

67. Titus, M.A. (2018). Myosin-driven intracellular transport. Cold Spring Harb. Perspect. Biol. 10, a021972.

68. Hegan, P.S., Giral, H., Levi, M., and Mooseker, M.S. (2012). Myosin VI is required for maintenance of brush border structure, composition, and membrane trafficking functions in the intestinal epithelial cell. Cytoskeleton 69, 235-251.

69. Hegan, P.S., Kravtsov, D.V., Caputo, C., Egan, M.E., Ameen, N.A., and Mooseker, M.S. (2015). Restoration of cytoskeletal and membrane tethering defects but not defects in membrane trafficking in the intestinal brush border of mice lacking both myosin la and myosin VI. Cytoskeleton 72, 455-476.

70. Crawley, S.W., Shifrin, D.A., Grega-Larson, N.E., McConnell, R.E., Benesh, A.E., Mao, S., Zheng, Y., Zheng, Q.Y., Nam, K.T., Millis, B.A., et al. (2014). Intestinal brush border assembly driven by protocadherin-based intermicrovillar adhesion. Cell 157, 433-446.

71. Weck, M.L., Crawley, S.W., Stone, C.R., and Tyska, M.J. (2016). Myosin-7b promotes distal tip localization of the intermicrovillar adhesion complex. Curr. Biol. 26, 2717-2728.

72. Choi, M.S., Graves, M.J., Matoo, S., Storad, Z.A., El Sheikh Idris, R.A., Weck, M.L., Smith, Z.B., Tyska, M.J., and Crawley, S.W. (2020). The small EF-hand protein CALML4 functions as a critical myosin light chain within the intermicrovillar adhesion complex. J. Biol. Chem. 295, 9281-9296.

73. Crawley, S.W., Weck, M.L., Grega-Larson, N.E., Shifrin, D.A., and Tyska, M.J. (2016). ANKS4B Is essential for intermicrovillar adhesion complex formation. Dev. Cell 36, 190200. 
74. Li, J., He, Y., Lu, Q., and Zhang, M. (2016). Mechanistic basis of organization of the harmonin/USH1C-mediated brush border microvilli tip-link complex. Dev. Cell 36, 179189.

75. Li, J., He, Y., Weck, M.L., Lu, Q., Tyska, M.J., and Zhang, M. (2017). Structure of Myo7b/USH1C complex suggests a general PDZ domain binding mode by MyTH4-FERM myosins. Proc. Natl. Acad. Sci. USA 114, E3776-E3785.

76. Yu, I.M., Planelles-Herrero, V.J., Sourigues, Y., Moussaoui, D., Sirkia, H., Kikuti, C., Stroebel, D., Titus, M.A., and Houdusse, A. (2017). Myosin 7 and its adaptors link cadherins to actin. Nat. Commun. 8, 15864.

77. He, Y., Li, J., and Zhang, M. (2019). Myosin VII, USH1C, and ANKS4B or USH1G together form condensed molecular assembly via liquid-liquid phase separation. Cell Rep. 29, 974-986.e4.

78. Weck, M.L., Crawley, S.W., and Tyska, M.J. (2020). A heterologous in-cell assay for investigating intermicrovillar adhesion complex interactions reveals a novel protrusion length-matching mechanism. J. Biol. Chem. 295, 16191-16206.

79. Henn, A., and De La Cruz, E.M. (2005). Vertebrate myosin VIIb is a high duty ratio motor adapted for generating and maintaining tension. J. Biol. Chem. 280, 39665-39676.

80. Chinowsky, C.R., Pinette, J.A., Meenderink, L.M., Lau, K.S., and Tyska, M.J. (2020). Nonmuscle myosin-2 contractility-dependent actin turnover limits the length of epithelial microvilli. Mol. Biol. Cell 31, 2803-2815.

81. Ebrahim, S., Fujita, T., Millis, B.A., Kozin, E., Ma, X., Kawamoto, S., Baird, M.A., Davidson, M., Yonemura, S., Hisa, Y., et al. (2013). NMIl forms a contractile transcellular sarcomeric network to regulate apical cell junctions and tissue geometry. Curr. Biol. 23, 731-736.

82. Pinette, J.A., Mao, S., Millis, B.A., Krystofiak, E.S., Faust, J.J., and Tyska, M.J. (2019). Brush border protocadherin CDHR2 promotes the elongation and maximized packing of microvilli in vivo. Mol. Biol. Cell 30, 108-118.

83. Rzadzinska, A.K., Schneider, M.E., Davies, C., Riordan, G.P., and Kachar, B. (2004). An actin molecular treadmill and myosins maintain stereocilia functional architecture and self-renewal. J. Cell Biol. 164, 887-897. 
84. McGrath, J., Roy, P., and Perrin, B.J. (2017). Stereocilia morphogenesis and maintenance through regulation of actin stability. Semin. Cell Dev. Biol. 65, 88-95.

85. Richardson, G.P., and Petit, C. (2019). Hair-bundle links: genetics as the gateway to function. Cold Spring Harb. Perspect. Med. 9, a033142.

86. Tarchini, B., and Lu, X. (2019). New insights into regulation and function of planar polarity in the inner ear. Neurosci. Lett. 709, 134373.

87. Krey, J.F., Chatterjee, P., Dumont, R.A., O'Sullivan, M., Choi, D., Bird, J.E., and BarrGillespie, P.G. (2020). Mechanotransduction-dependent control of stereocilia dimensions and row identity in inner hair cells. Curr. Biol. 30, 442-454.e7.

88. Velez-Ortega, A.C., and Frolenkov, G.I. (2019). Building and repairing the stereocilia cytoskeleton in mammalian auditory hair cells. Hear. Res. 376, 47-57.

89. Elliott, K.L., Fritzsch, B., and Duncan, J.S. (2018). Evolutionary and developmental biology provide insights into the regeneration of Organ of Corti hair cells. Front. Cell Neurosci. 12, 252.

90. Maoileidigh, D.O., and Ricci, A.J. (2019). A bundle of mechanisms: inner-ear haircell mechanotransduction. Trends Neurosci. 42, 221-236.

91. Gillespie, P.G., and Muller, U. (2009). Mechanotransduction by hair cells: models, molecules, and mechanisms. Cell 139, 33-44.

92. Shin, J.B., Krey, J.F., Hassan, A., Metlagel, Z., Tauscher, A.N., Pagana, J.M., Sherman, N.E., Jeffery, E.D., Spinelli, K.J., Zhao, H., et al. (2013). Molecular architecture of the chick vestibular hair bundle. Nat. Neurosci. 16, 365-374.

93. Grati, M., and Kachar, B. (2011). Myosin VIla and sans localization at stereocilia upper tip-link density implicates these Usher syndrome proteins in mechanotransduction. Proc. Natl. Acad. Sci. USA 108, 11476-11481.

94. Kros, C.J., Marcotti, W., van Netten, S.M., Self, T.J., Libby, R.T., Brown, S.D.M., Richardson, G.P., and Steel, K.P. (2002). Reduced climbing and increased slipping adaptation in cochlear hair cells of mice with Myo7a mutations. Nat. Neurosci. 5, 41-47.

95. Li, S., Mecca, A., Kim, J., Caprara, G.A., Wagner, E.L., Du, T.T., Petrov, L., Xu, W., Cui, R., Rebustini, I.T., et al. (2020). Myosin-VIla is expressed in multiple isoforms and 
essential for tensioning the hair cell mechanotransduction complex. Nat. Commun. 11, 2066.

96. Laakso, J.M., Lewis, J.H., Shuman, H., and Ostap, E.M. (2010). Control of myosin-I force sensing by alternative splicing. Proc. Natl. Acad. Sci. USA 107, 698-702.

97. Robert-Paganin, J., Robblee, J.P., Auguin, D., Blake, T.C.A., Bookwalter, C.S., Krementsova, E.B., Moussaoui, D., Previs, M.J., Jousset, G., Baum, J., et al. (2019). Plasmodium myosin A drives parasite invasion by an atypical force generating mechanism. Nat. Commun. 10, 3286.

98. Goodyear, R., and Richardson, G. (1999). The ankle-link antigen: an epitope sensitive to calcium chelation associated with the hair-cell surface and the calycal processes of photoreceptors. J. Neurosci. 19, 3761-3772.

99. Boëda, B., El-Amraoui, A., Bahloul, A., Goodyear, R., Daviet, L., Blanchard, S., Perfettini, I., Fath, K.R., Shorte, S., Reiners, J., et al. (2002). Myosin VIla, harmonin and cadherin 23, three Usher 1 gene products that cooperate to shape the sensory hair bundle. EMBO J. 21,6689-6699.

100. Morgan, C.P., Krey, J.F., Grati, M., Zhao, B., Fallen, S., Kannan-Sundhari, A., Liu, X.Z., Choi, D., Müller, U., and Barr-Gillespie, P.G. (2016). PDZD7-MYO7A complex identified in enriched stereocilia membranes. eLife 5, e18312.

101. Gibson, F., Walsh, J., Mburu, P., Varela, A., Brown, K.A., Antonio, M., Beisel, K.W., Steel, K.P., and Brown, S.D.M. (1995). A type VII myosin encoded by the mouse deafness gene shaker-1. Nature 374, 62-64.

102. Weil, D., Blanchard, S., Kaplan, J., Guliford, P., Gibson, F., Walsh, J., Mburu, P., Varela, A., Levilliers, J., Weston, M.D., et al. (1995). Defective myosin VIIA gene responsible for Usher syndrome type 1B. Nature 374,60-61.

103. Nicolson, T., Rüsch, A., Friedrich, R.W., Granato, M., Ruppersberg, J.P., and Nüsslein-Volhard, C. (1999). Genetic analysis of vertebrate sensory hair cell mechanosensation: the zebrafish circler mutants. Neuron 20, 271-283.

104. Self, T., Mahony, M., Fleming, J., Walsh, J., Brown, S.D.M., and Steel, K.P. (1998). Shaker-1 mutations reveal roles for myosin VIIA in both development and function of cochlear hair cells. Development 125, 557-566. 
105. Pan, L., Yan, J., Wu, L., and Zhang, M. (2009). Assembling stable hair cell tip link complex via multidentate interactions between harmonin and cadherin 23. Proc. Natl. Acad. Sci. USA 106, 5575-5580.

106. Wu, L., Pan, L., Wei, Z., and Zhang, M. (2011). Structure of MyTH4-FERM domains in myosin VIla tail bound to cargo. Science 331, 757-760.

107. Bahloul, A., Pepermans, E., Raynal, B., Wolff, N., Cordier, F., England, P., Nouaille, S., Baron, B., El-Amraoui, A., Hardelin, J.P., et al. (2017). Conformational switch of harmonin, a submembrane scaffold protein of the hair cell mechanoelectrical transduction machinery. FEBS Lett. 591, 2299-2310.

108. Grillet, N., Xiong, W., Reynolds, A., Kazmierczak, P., Sato, T., Lillo, C., Dumont, R.A., Hintermann, E., Sczaniecka, A., Schwander, M., et al. (2009). Harmonin mutations cause mechanotransduction defects in cochlear hair cells. Neuron 62, 375-387.

109. Michalski, N., Michel, V., Caberlotto, E., Lefèvre, G.M., van Aken, A.F., Tinevez, J.Y., Bizard, E., Houbron, C., Weil, D., Hardelin, J.P., et al. (2009). Harmonin-b, an actin-binding scaffold protein, is involved in the adaptation of mechanoelectrical transduction by sensory hair cells. Pflugers Arch. 459, 115-130.

110. Verpy, E., Leibovici, M., Zwaenepoel, I., Liu, X.Z., Gal, A., Salem, N., Mansour, A., Blanchard, S., Kobayashi, I., Keats, B.J., et al. (2000). A defect in harmonin, a PDZ domaincontaining protein expressed in the inner ear sensory hair cells, underlies Usher syndrome type 1C. Nat. Genet. 26, 51-55.

111. Yan, J., Pan, L., Chen, X., Wu, L., and Zhang, M. (2010). The structure of the harmonin/sans complex reveals an unexpected interaction mode of the two Usher syndrome proteins. Proc. Natl. Acad. Sci. USA 107, 4040-4045.

112. Krey, J.F., and Barr-Gillespie, P.G. (2019). Molecular Composition of Vestibular Hair Bundles. Cold Spring Harb. Perspect. Med. 9, a033209.

113. Zhu, Y., Scheibinger, M., Ellwanger, D.C., Krey, J.F., Choi, D., Kelly, R.T., Heller, S., and Barr-Gillespie, P.G. (2019). Single-cell proteomics reveals changes in expression during hair-cell development. eLife 8, e50777.

114. Sakai, T., Jung, H.S., Sato, O., Yamada, M.D., You, D.J., Ikebe, R., and Ikebe, M. (2015). Structure and regulation of the movement of human myosin VIIA. J. Biol. Chem. 290, 17587-17598. 
115. Sakai, T., Umeki, N., Ikebe, R., and Ikebe, M. (2011). Cargo binding activates myosin VIIA motor function in cells. Proc. Natl. Acad. Sci. USA 108, 7028-7033.

116. Michalski, N., Michel, V., Bahloul, A., Lefèvre, G., Barral, J., Yagi, H., Chardenoux, S., Weil, D., Martin, P., Hardelin, J.P., et al. (2007). Molecular characterization of the anklelink complex in cochlear hair cells and its role in the hair bundle functioning. J. Neurosci. $27,6478-6488$.

117. Zou, J., Chen, Q., Almishaal, A., Mathur, P.D., Zheng, T., Tian, C., Zheng, Q.Y., and Yang, J. (2017). The roles of USH1 proteins and PDZ domain-containing USH proteins in USH2 complex integrity in cochlear hair cells. Hum. Mol. Genet. 26, 624-636.

118. Zou, J., Mathur, P.D., Zheng, T., Wang, Y., Almishaal, A., Park, A.H., and Yang, J. (2015). Individual USH2 proteins make distinct contributions to the ankle link complex during development of the mouse cochlear stereociliary bundle. Hum. Mol. Genet. 24, 6944-6957.

119. Ebermann, I., Phillips, J.B., Liebau, M.C., Koenekoop, R.K., Schermer, B., Lopez, I., Schäfer, E., Roux, A.F., Dafinger, C., Bernd, A., et al. (2010). PDZD7 is a modifier of retinal disease and a contributor to digenic Usher syndrome. J. Clin. Invest. 120, 1812-1823.

120. Mburu, P., Mustapha, M., Varela, A., Weil, D., El-Amraoui, A., Holme, R.H., Rump, A., Hardisty, R.E., Blanchard, S., Coimbra, R.S., et al. (2003). Defects in whirlin, a PDZ domain molecule involved in stereocilia elongation, cause deafness in the whirler mouse and families with DFNB31. Nat. Genet. 34, 421-428.

121. Küssel-Andermann, P., El-Amraoui, A., Safieddine, S., Nouaille, S., Perfettini, I., Lecuit, M., Cossart, P., Wolfrum, U., and Petit, C. (2000). Vezatin, a novel transmembrane protein, bridges myosin VIIA to the cadherin-catenins complex. EMBO J. 19,6020-6029.

122. Avraham, K.B., Hasson, T., Steel, K.P., Kingsley, D.M., Russell, L.B., Mooseker, M.S., Copeland, N.G., and Jenkins, N.A. (1995). The mouse Snell's waltzer deafness gene encodes an unconventional myosin required for structural integrity of inner ear hair cells. Nat. Genet. 11, 369-375.

123. Hasson, T., Gillespie, P.G., Garcia, J.A., MacDonald, R.B., Zhao, Y., Yee, A.G., Mooseker, M.S., and Corey, D.P. (1997). Unconventional myosins in inner-ear sensory epithelia. J. Cell Biol. 137, 1287-1307. 
124. Self, T., Sobe, T., Copeland, N.G., Jenkins, N.A., Avraham, K.B., and Steel, K.P. (1999). Role of myosin VI in the differentiation of cochlear hair cells. Dev. Biol. 214, 331341.

125. Sakaguchi, H., Tokita, J., Naoz, M., Bowen-Pope, D., Gov, N.S., and Kachar, B. (2008). Dynamic compartmentalization of protein tyrosine phosphatase receptor $Q$ at the proximal end of stereocilia: implication of myosin VI-based transport. Cell Motil. Cytoskeleton 65, 528-538.

126. Salles, F.T., Andrade, L.R., Tanda, S., Grati, M., Plona, K.L., Gagnon, L.H., Johnson, K.R., Kachar, B., and Berryman, M.A. (2014). CLIC5 stabilizes membrane-actin filament linkages at the base of hair cell stereocilia in a molecular complex with radixin, taperin, and myosin VI. Cytoskeleton 71, 61-78.

127. Seki, Y., Miyasaka, Y., Suzuki, S., Wada, K., Yasuda, S.P., Matsuoka, K., Ohshiba, Y., Endo, K., Ishii, R., Shitara, H., et al. (2017). A novel splice site mutation of myosin VI in mice leads to stereociliary fusion caused by disruption of actin networks in the apical region of inner ear hair cells. PLoS One 12, e0183477.

128. Cirilo, J.A., Gunther, L.K., and Yengo, C.M. (2021). Functional role of class III myosins in hair cells. Front. Cell Dev. Biol. 9, 643856.

129. Quintero, O.A., Unrath, W.C., Stevens, S.M.J., Manor, U., Kachar, B., and Yengo, C.M. (2013). Myosin 3A kinase activity is regulated by phosphorylation of the kinase domain activation loop. J. Biol. Chem. 288, 37126-37137.

130. Raval, M.H., Quintero, O.A., Weck, M.L., Unrath, W.C., Gallagher, J.W., Cui, R., Kachar, B., Tyska, M.J., and Yengo, C.M. (2016). Impact of the motor and tail domains of class III myosins on regulating the formation and elongation of actin protrusions. J. Biol. Chem. 291, 22781-22792.

131. Salles, F.T., Merritt, R.C.J., Manor, U., Dougherty, G.W., Sousa, A.D., Moore, J.E., Yengo, C.M., Dose, A.C., and Kachar, B. (2009). Myosin Illa boosts elongation of stereocilia by transporting espin 1 to the plus ends of actin filaments. Nat. Cell Biol. 11, 443-350.

132. Erickson, F.L., Corsa, A.C., Dosé, A., and Burnside, B. (2003). Localization of a class III myosin to filopodia tips in transfected HeLa cells. Mol. Biol. Cell 14, 4173-4180. 
133. Merritt, R.C., Manor, U., Salles, F.T., Grati, M., Dosé, A.C., Unrath, W.C., Quintero, O.A., Yengo, C.M., and Kachar, B. (2012). Myosin IIIB uses an actin-binding motif in Its espin-1 cargo to reach the tips of actin protrusions. Curr. Biol. 22, 320-325.

134. Liu, H., Li, J., Raval, M.H., Yao, N., Deng, X., Lu, Q., Nie, S., Feng, W., Wan, J., Yengo, C.M. et al. (2016). Myosin III-mediated cross-linking and stimulation of actin bundling activity of Espin. eLife 5, e12856.

135. Ebrahim, S., Avenarius, M.R., Grati, M., Krey, J.F., Windsor, A.M., Sousa, A.D., Ballesteros, A., Cui, R., Millis, B.A., Salles, F.T., et al. (2016). Stereocilia-staircase spacing is influenced by myosin III motors and their cargos espin-1 and espin-like. Nat. Commun. $7,10833$.

136. Schneider, M.E., Dose, A.C., Salles, F.T., Chang, W., Erickson, F.L., Burnside, B., and Kachar, B. (2006). A new compartment at stereocilia tips defined by spatial and temporal patterns of myosin IIla expression. J. Neurosci. 26, 10243-10252.

137. Lelli, A., Michel, V., Boutet de Monvel, J., Cortese, M., Bosch-Grau, M., Aghaie, A., Perfettini, I., Dupont, T., Avan, P., El-Amraoui, A., et al. (2016). Class III myosins shape the auditory hair bundles by limiting microvilli and stereocilia growth. J. Cell Biol. 212, 231244.

138. Walsh, T., Walsh, V., Vreugde, S., Hertzano, R., Shahin, H., Haika, H., Lee, M.K., Kanaan, M., King, M.C., and Avraham, K.B. (2002). From flie's eyes to our ears: mutations in a human class III myosin cause progressive nonsyndromic hearing loss DFNB30. Proc. Natl. Acad. Sci. USA 99, 7518-7523.

139. Walsh, V.L., Raviv, D., Dror, A.A., Shahin, H., Walsh, T., Kanaan, M.N., Avraham, K.B., and King, M.C. (2010). A mouse model for human hearing loss DFNB30 due to loss of function of myosin IIIA. Mamm. Genome 22, 170-177.

140. Belyantseva, I.A., Boger, E.T., and Friedman, T.B. (2003). Myosin XVa localizes to the tips of inner ear sensory cell stereocilia and is essential for staircase formation of the hair bundle. Proc. Natl. Acad. Sci. USA 100, 13958-13963.

141. Probst, F.J., Fridell, R.A., Raphael, Y., Saunders, T.L., Wang, A., Liang, Y., Morell, R.J., Touchman, J.W., Lyons, R.H., Noben-Trauth, K., et al. (1998). Correction of deafness in shaker-2 mice by an unconventional myosin in a BAC transgene. Science. 280, 14441447. 
142. Bird, J.E., Barzik, M., Drummond, M.C., Sutton, D.C., Goodman, S.M., Morozko, E.L., Cole, S.M., Boukhvalova, A.K., Skidmore, J., Syam, D., et al. (2017). Harnessing molecular motors for nanoscale pulldown in live cells. Mol. Biol. Cell 28, 463-475.

143. Jiang, F., Takagi, Y., Shams, A., Heissler, S.M., Friedman, T.B., Sellers, J.R., and Bird, J.E. (2021). The ATPase mechanism of myosin 15, the molecular motor mutated in DFNB3 human deafness. J. Biol. Chem. 296, 100243.

144. Belyantseva, I.A., Boger, E.T., Naz, S., Frolenkov, G.I., Sellers, J.R., Ahmed, Z.M., Griffith, A.J., and Friedman, T.B. (2005). Myosin-XVa is required for tip localization of whirlin and differential elongation of hair-cell stereocilia. Nat. Cell Biol. 7, 148-156.

145. Liang, Y., Wang, A., Belyantseva, I.A., Anderson, D.W., Probst, F.J., Barber, T.D., Miller, W., Touchman, J.W., Jin, L., Sullivan, S.L., et al. (1999). Characterization of the human and mouse unconventional myosin XV genes responsible for hereditary deafness DFNB3 and shaker 2. Genomics 61, 243-258.

146. Fang, Q., Indzhykulian, A.A., Mustapha, M., Riordan, G.P., Dolan, D.F., Friedman, T.B., Belyantseva, I.A., Frolenkov, G.I., Camper, S.A., and Bird, J.E. (2015). The 133-kDa Nterminal domain enables myosin 15 to maintain mechanotransducing stereocilia and is essential for hearing. elife 4 , e08627.

147. Delprat, B., Michel, V., Goodyear, R., Yamasaki, Y., Michalski, N., El-Amraoui, A., Perfettini, I., Legrain, P., Richardson, G., Hardelin, J.P., et al. (2005). Myosin XVa and whirlin, two deafness gene products required for hair bundle growth, are located at the stereocilia tips and interact directly. Hum. Mol. Genet. 14, 401-410.

148. Manor, U., Disanza, A., Grati, M., Andrade, L., Lin, H., Di Fiore, P.P., Scita, G., and Kachar, B. (2011). Regulation of stereocilia length by myosin XVa and whirlin depends on the actin-regulatory protein Eps8. Curr. Biol. 21, 167-172.

149. Mauriac, S.A., Hien, Y.E., Bird, J.E., Carvalho, S.D., Peyroutou, R., Lee, S.C., Moreau, M.M., Blanc, J.M., Geyser, A., Medina, C., et al. (2017). Defective Gpsm2/G $\alpha^{\text {i3 }}$ signalling disrupts stereocilia development and growth cone actin dynamics in ChudleyMcCullough syndrome. Nat. Commun. 8, 14907.

150. Tadenev, A.L.D., Akturk, A., Devanney, N., Mathur, P.D., Clark, A.M., Yang, J., and Tarchini, B. (2019). GPSM2-GNAI specifies the tallest stereocilia and defines hair bundle row identity. Curr. Biol. 29, 921-934.e4. 
151. Lin, L., Shi, Y., Wang, M., Wang, C., Lu, Q., Zhu, J., and Zhang, R. (2021). Phase separation-mediated condensation of Whirlin-Myo15-Eps8 stereocilia tip complex. Cell Rep. 34, 108770.

152. Frolenkov, G.I., Belyantseva, I.A., Friedman, T.B., and Griffith, A.J. (2004). Genetic insights into the morphogenesis of inner ear hair cells. Nat. Rev. Genet. 5, 489-498.

153. Hadi, S., Alexander, A.J., Vélez-Ortega, A.C., and Frolenkov, G.I. (2020). Myosin$\mathrm{XVa}$ controls both staircase architecture and diameter gradation of stereocilia rows in the auditory hair cell bundles. J. Assoc. Res. Otolaryngol. 21, 121-135.

154. Biyasheva, A., Svitkina, T., Kunda, P., Baum, B., and Borisy, G. (2004). Cascade pathway of filopodia formation downstream of SCAR. J. Cell Sci. 117, 837-848.

155. Lefèvre, G., Michel, V., Weil, D., Lepelletier, L., Bizard, E., Wolfrum, U., Hardelin, J. P., and Petit, C. (2008). A core cochlear phenotype in USH1 mouse mutants implicates fibrous links of the hair bundle in its cohesion, orientation and differential growth.

Development 135, 1427-1437. 
Figure 1.
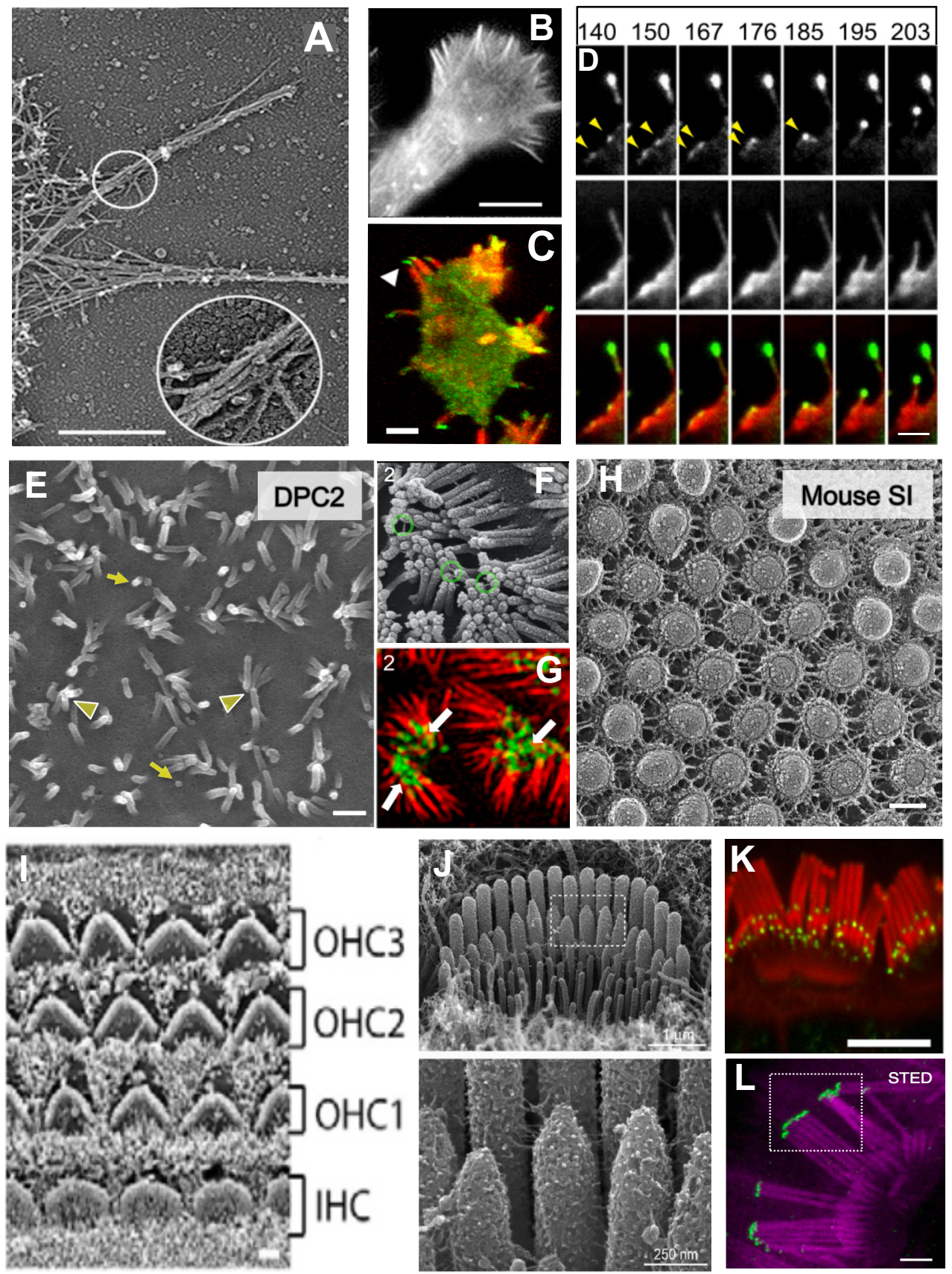


\section{Filopodial Myosins}

MYO10

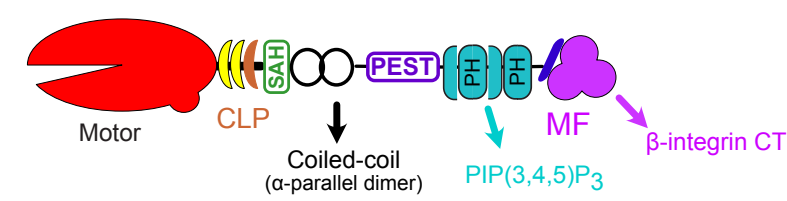

DdMyo7

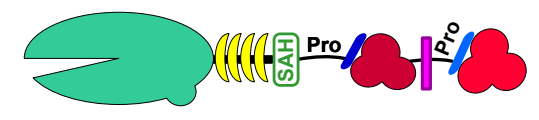

MYH9

(Myo2A)

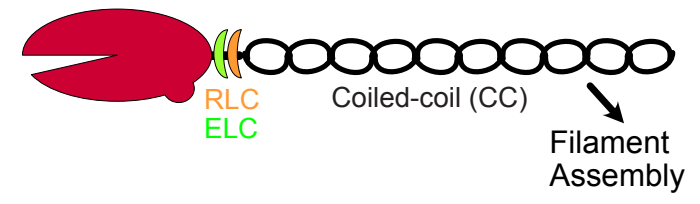

\section{Microvillar Myosins}

MY01A

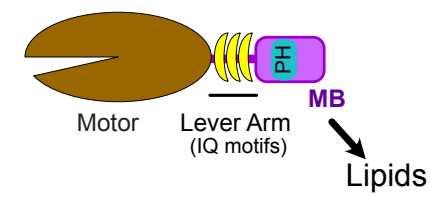

MYH14 (Myo2C)

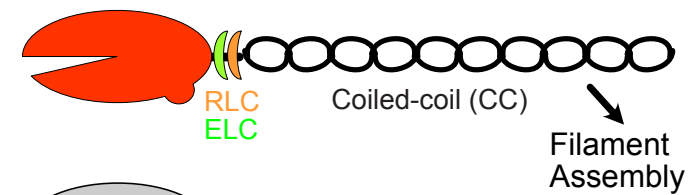

MYO6

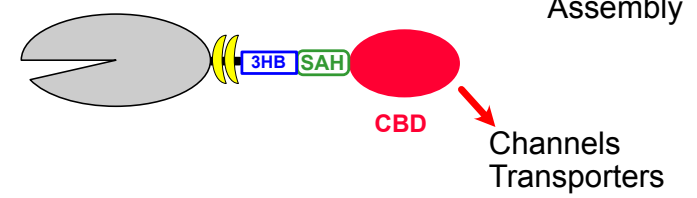

MYO7B

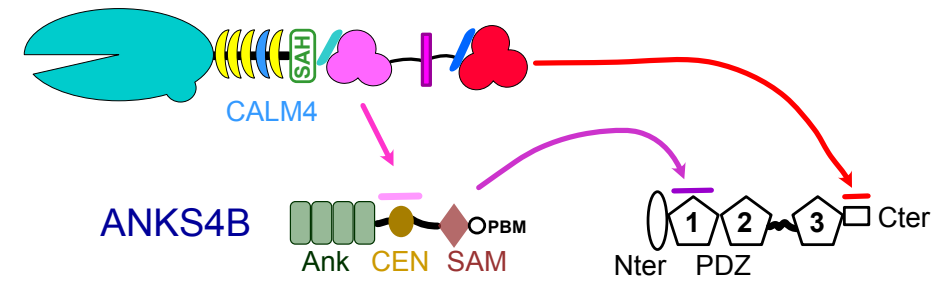

Harmonin

\section{Stereocilia Myosins}

MYO1C

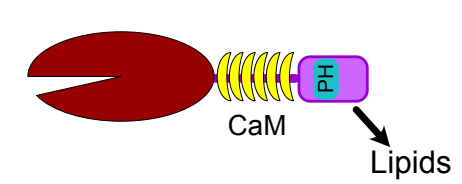

MYO3A

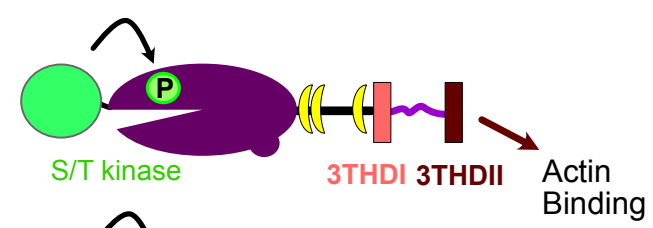

MYO3B

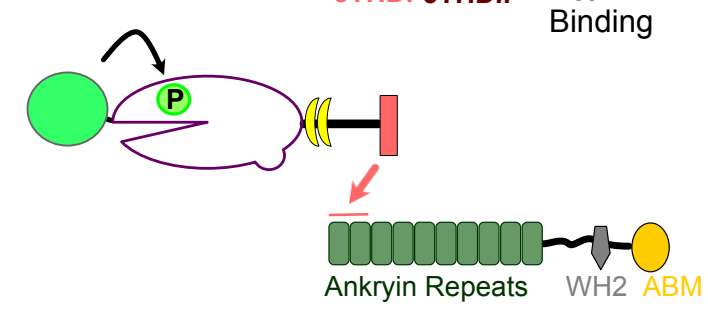

\section{Espin1}

MYO6

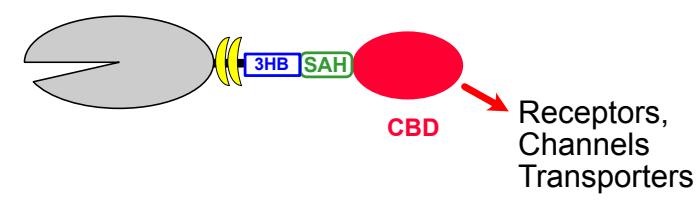

MYO7A

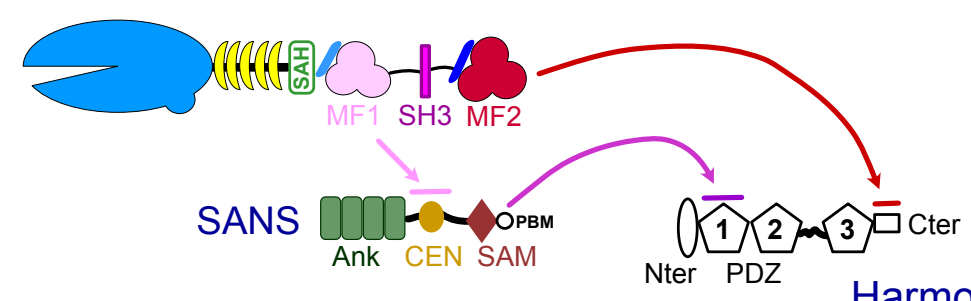

MYO15A

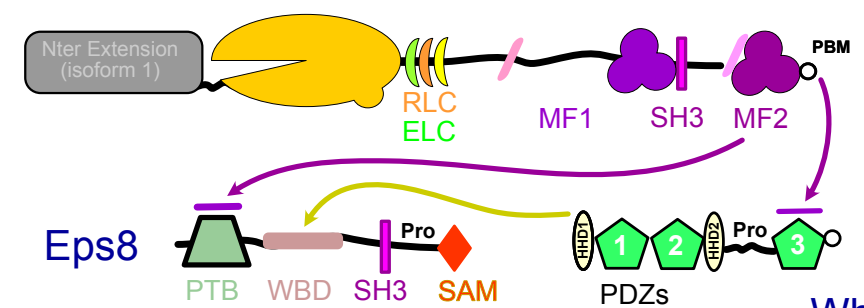

Whirlin 
A.

(6)

Translocate up growing

filament

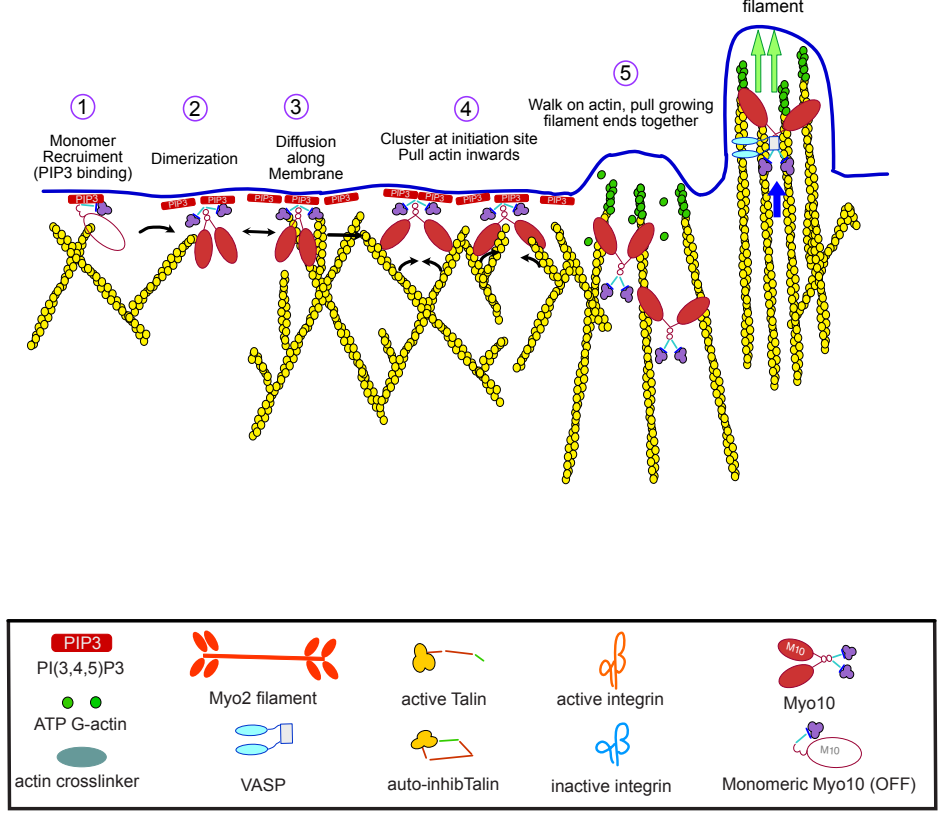

B.

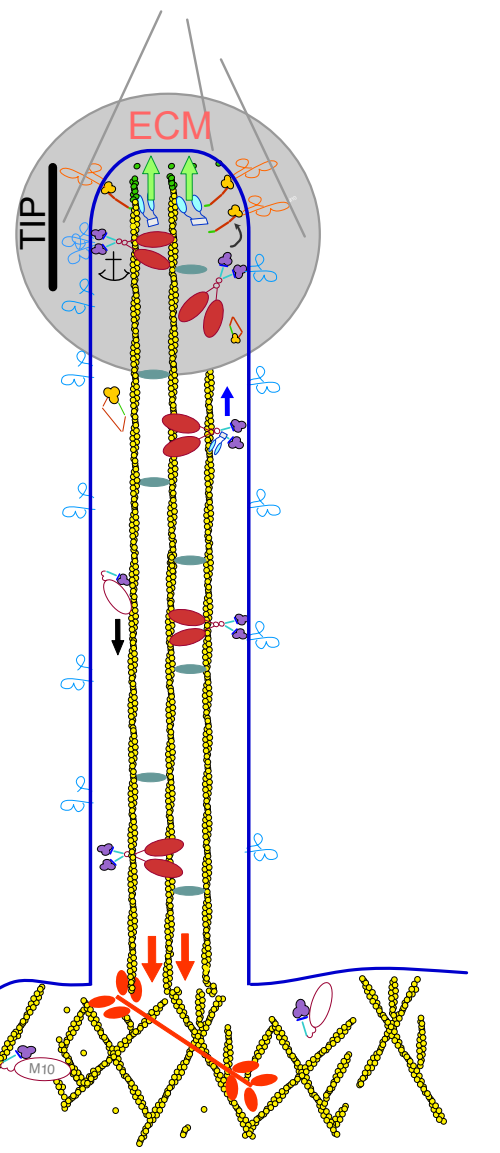



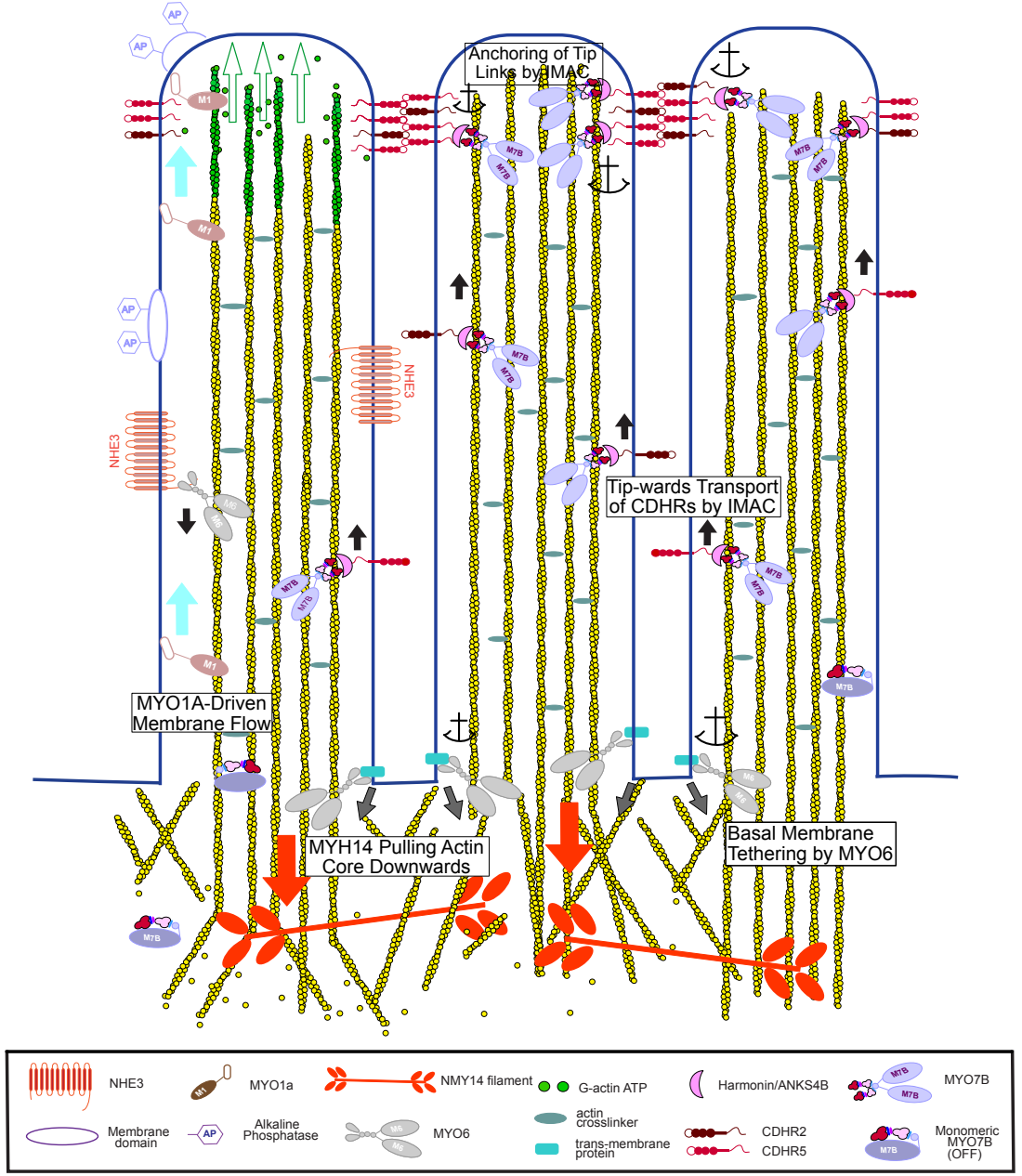


\section{Figure 5.}

Postnatal 4.5

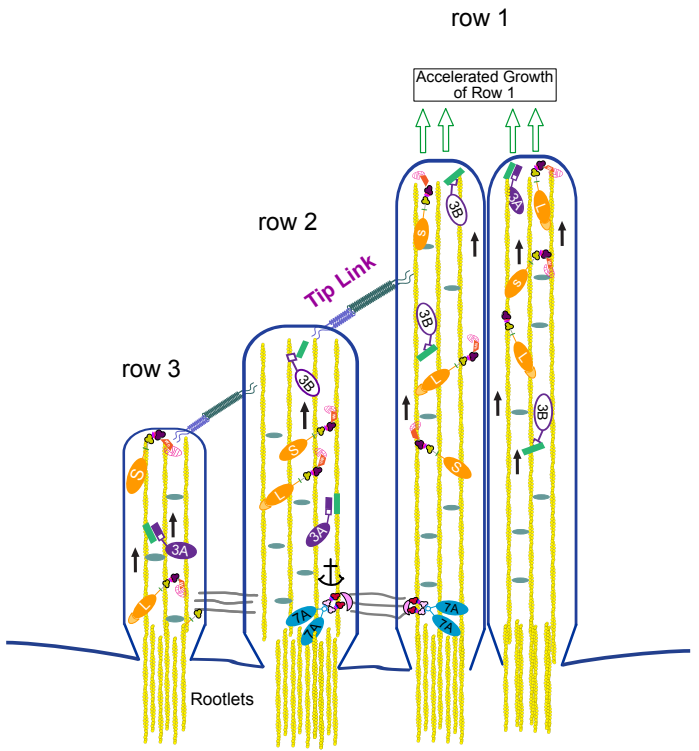

\section{Postnatal 15 \\ row 1}

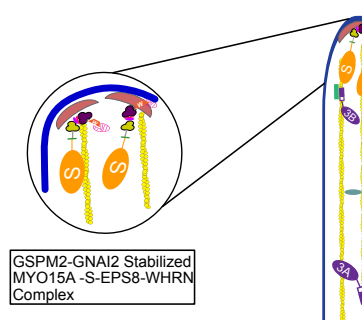

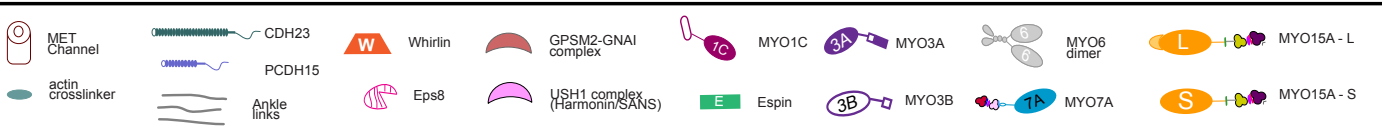

